\title{
Permutations, Moments, Measures
}

\author{
Natasha Blitvić* \\ Department of Mathematics and Statistics \\ Lancaster University, UK \\ natasha.blitvic@lancaster.ac.uk
}

\author{
Einar Steingrímsson ${ }^{\dagger}$ \\ Department of Mathematics and Statistics \\ University of Strathclyde, UK \\ einar@alum.mit.edu
}

September 11, 2020

\begin{abstract}
Which combinatorial sequences correspond to moments of probability measures on the real line? We present a generating function, in the form of a continued fraction, for a fourteenparameter family of such sequences and interpret these in terms of combinatorial statistics on the symmetric groups. Special cases include several classical and noncommutative probability laws, along with a substantial subset of the orthogonalizing measures in the $q$-Askey scheme, now given a new combinatorial interpretation in terms of elementary permutation statistics. This framework further captures a variety of interesting combinatorial sequences including, notably, the moment sequences associated to distributions of the numbers of occurrences of (classical and vincular) permutation patterns of length three. This connection between pattern avoidance and broader ideas in classical and noncommutative probability is among several intriguing new corollaries, which generalize and unify results previously appearing in the literature, while opening up new lines of inquiry.

The fourteen combinatorial statistics further generalize to signed and colored permutations, and, as an infinite family of statistics, to the $k$-arrangements: permutations with $k$-colored fixed points, introduced here along with several related results and conjectures.
\end{abstract}

\section{Introduction}

By describing the distributions of random objects in terms of their moment sequences, a number of fundamental probability laws are seen to be equivalent to key constructions in combinatorics. A well-known example is the semicircle law [Wig55] whose ubiquity in random matrix theory and free probability is paralleled by the pervasiveness of its moments, the Catalan numbers, in the enumerative world. Similarly, the moments of the Poisson and Gaussian laws enumerate, respectively, set partitions and perfect matchings of sets, while the 'noncommutative analogues' of these probability laws are given by combinatorial refinements of the aforementioned moment sequences [BS91, Ans01, Bli12, BEH15, Ejs]. This probabilistic interpretation further extends to combinatorial statistics on set partitions, symmetric groups and other Coxeter groups [BS94, BEH17].

The classical theorem of Hamburger [Ham20] provides a complete characterization of moment sequences associated to (positive Borel) measures on the real line. Namely, given some real-valued

${ }^{*}$ This work was completed while the author was an Academic Guest at ETH Zürich.

${ }^{\dagger}$ Partially supported by a Leverhulme Research Fellowship. 
sequence $\left(m_{n}\right)_{n \geq 0}$, the existence of a measure $\mu$ with the property that

$$
\int_{\mathbb{R}} x^{n} d \mu(x)=m_{n}
$$

is equivalent to the positive semidefiniteness of the Hankel matrices $\left(m_{i+j}\right)_{0 \leq i, j \leq n}$ for all $n \in \mathbb{N}_{0}$. (The measure $\mu$ is of course a probability measure whenever $m_{0}=1$.) Unfortunately, establishing the positivity of the Hankel matrices is a difficult combinatorial problem. Rather, showing that a given combinatorial sequence is a moment sequence is typically approached by other means, including explicitly writing down the corresponding measure (see e.g. [Mło10, Mło12, MP14, MK15, MP18, Sok18]).

On the whole, the integer sequences that are positive definite (in the preceding sense) are relatively few, but their class seems to include surprisingly many classical sequences. Rather than considering such problems in isolation, we are interested in general principles that may help elucidate the link between combinatorial structure and positivity.

In this paper, we introduce a fourteen-parameter class of combinatorial sequences which are moments of measures on the real line. Our central object is the following continued fraction.

Definition 1. For parameters $a, b, c, d, f, g, h, \ell, p, r, s, t, u, w \in \mathbb{R}$, let

$$
\mathcal{C}(z)=\mathcal{C}_{a, b, c, d, f, g, h, \ell, p, r, s, t, u, w}(z):=\frac{1}{1-\alpha_{0} z-\frac{\beta_{1} z^{2}}{1-\alpha_{1} z-\frac{\beta_{2} z^{2}}{\ddots}}}
$$

with

$$
\alpha_{n}=u \cdot w^{n}+s[n]_{a, b}+t[n]_{f, g}, \quad \beta_{n}=p r[n]_{c, d}[n]_{h, \ell} .
$$

(Above, we follow the standard convention by letting $[n]_{x, y}:=x^{n-1}+x^{n-2} y+\cdots+x y^{n-2}+y^{n-1}$ for $n \in \mathbb{N}$, with $[0]_{x, y}:=0$.)

By our main theorem (Theorem 1, next section), the continued fraction $\mathcal{C}$ has a natural combinatorial interpretation in terms of fourteen elementary combinatorial statistics on permutations, to be defined in detail in the next section. Except for the traditionally defined fixed points, these are all based on excedances, that is, integers $i \in[n]:=\{1,2, \ldots, n\}$ such that $\sigma(i)>i$, anti-excedances, $\sigma(i)<i$, and inversions among letters of $\sigma$, that is, pairs $(i, j)$ such that $i<j$ and $\sigma(i)>\sigma(j)$. We distinguish between linked and non-linked excedances (an intuitive geometric property, defined shortly) and anti-excedances, respectively, as well as between inversions and non-inversions associated to these. An aspect of the continued fraction $\mathcal{C}$ worth highlighting is the systematic nature of its combinatorial interpretation. Namely, the fourteen combinatorial statistics characterize the action of $\sigma \in \mathcal{S}_{n}$ on each element of $[n]:=\{1,2, \ldots, n\}$ by placing each pair $i \mapsto \sigma(i)$ into one of the five aforementioned excedance-based classes, with the relations between the elements in each class (and in one case between classes) captured in terms of inversions and non-inversions. As a result, it becomes relatively straightforward to understand, on a combinatorial level, the appearance in specializations of $\mathcal{C}$ of the combinatorial families given in Table 1.

Continued fraction expansions of ordinary generating functions commonly appear in combinatorial theory, particularly in relation to labeled Motzkin paths [Fla80]. As such, the continued fraction $\mathcal{C}$ generalizes aspects of several important papers connecting continued fractions, Motzkin paths and statistics on permutations and other combinatorial structures, such as [FV79, Fla80, FZ90, Bia93, dMV94, Eli17]. Given a continued fraction associated with a well-studied combinatorial sequence, 
there are typically two approaches to arriving at a more general result. One consists of attempting to 'fold in' multiple related combinatorial statistics into one generating function, such as in e.g. [CSZ97, IKZ13, Eli17]. That is, given some multivariate generating function associated to several combinatorial sequences, the problem consists of identifying the continued fraction expansion of the said generating function. Most recently, Sokal and Zeng [SZ] have undertaken an impressive study of various ways in which statistics on permutations, set partitions and perfect matchings can be consolidated in this manner.

The other approach, taken presently, seeks to provide a more general scheme of generating functions. Here, the challenge is that of giving a natural combinatorial interpretation for some multiparameter continued fraction or, equivalently, for a multiparameter family of polynomials that are orthogonal with respect to some linear functional. The continued fractions studied may be associated with new schemes of orthogonal polynomials, such as [SS94, SS96, Ran98], or well-known ones, as in the particularly notable works of Corteel and Williams et al. [CW11, CSSW12] that provide a combinatorial interpretation for the Askey-Wilson family of $q$-hypergeometric orthogonal polynomials.

We find that the continued fraction $\mathcal{C}$ of Definition 1 includes a surprising number of interesting specializations of the Askey-Wilson polynomials, which appear as named families in the $q$-Askey scheme [KLS10]. In this manner, $\mathcal{C}$ ascribes a new and considerably more transparent combinatorial interpretation, in terms of elementary permutation statistics, to a substantial portion of the $q$-Askey scheme, while generalizing these families in a different direction. Furthermore, $\mathcal{C}$ extends the so-called octabasic Laguerre family of Simion and Stanton [SS94, SS96], also associated with combinatorial statistics on the symmetric groups. A crucial difference between our approach and that of Simion and Stanton is in the explicit accounting of the fixed points, which in prior work are combined with anti-excedances to form non-excedances. The result is a considerably greater degree of generality, admitting a wider array of interesting special cases, and a greater symmetry in the continued fraction. (Note that Simion and Stanton do not work with 'excedance based' statistics, but their statistics correspond, via a certain bijection, to such statistics, as further discussed in Section 4.4.) Our work also partially extends a sixteen-parameter framework of Randrianrivony [Ran98], itself another generalization of the work of Simion and Stanton. A different combinatorial interpretation of our fourteen-parameter continued fraction $\mathcal{C}$ can be obtained by specializing an eighteen-parameter one in a recent paper by Sokal and Zeng [SZ]. They accomplish this by 'mixing' statistics of two inherently different kinds, based respectively on excedances and 'records' (relative maxima and minima), and by also using refinements of the crossing and nesting statistics of Corteel [Cor07]. A detailed discussion of the similarities and differences between all these combinatorial approaches is found in Section 4.4.

The simplicity of our combinatorial interpretation of the continued fraction $\mathcal{C}$ particularly pays dividends when considering the corollaries and extensions of the main theorem (Theorem 1). In particular, simple generalizations of our continued fraction capture corresponding statistics on the hyperoctahedral groups (signed permutations) and certain unitary reflection groups (colored permutations). Statistics on the latter were introduced in [Ste94] and subsequently studied by various authors. In another direction, the separation of the fixed points in $\mathcal{C}$ leads naturally to a generalization capturing various statistics on the $k$-arrangements, introduced here, namely permutations with $k$-colored fixed points. These form an infinite family of combinatorial objects containing in a natural way the derangements (fixed-point free permutations), permutations and what previously have been called just arrangements by Comtet [Com74], which also coincide with Postnikov's definition of 'decorated permutations' [Pos]. We present formulas and an exponential generating function for the numbers of $k$-arrangements along with a few results on combinatorial statistics on these objects and several conjectures.

Most of the corollaries appearing in this paper are new. Those that have previously appeared in 
more specialized works gain clarity by being seen to follow from a more general principle, as well as a new dimension, through juxtaposition to new ideas. This general perspective also opens up new lines of inquiry. As previously mentioned, the combinatorial statistics naturally lead us to define the notion of a $k$-arrangement, which come with interesting properties, some of which are shown here and others conjectured. This combinatorial framework also raises a number of natural questions from the point of view of probability and analysis, as the measures arising in the present setting include a surprising number of well-studied probability laws which were previously not connected into a single framework. And at the interface of probability and combinatorics, the framework suggests that pattern avoidance, at least for some patterns, may be naturally interpreted in terms of moments of random variables (whether classical or noncommutative), proposing new ways of approaching the long-standing open problems in this arena.

This paper is organized as follows: In Section 2 we define the permutation statistics at the core of our constructions and present the main theorem (Theorem 1), which describes the connection between these statistics and the continued fraction $\mathcal{C}$. The correspondence relies on labeled Motzkin paths, using Flajolet's general correspondence.

In Section 3 we describe, in six subsections, the various results derived from the main theorem. Namely, we identify the parameter ranges for which a sequence arising from $\mathcal{C}$ is a moment sequence of a unique probability measure on the real line (Section 3.1). As previously mentioned, the family of moment sequences encompassed by $\mathcal{C}$ is surprisingly interesting, including several well-known classical laws as well as noncommutative central limits. Furthermore, the orthogonal polynomials associated with $\mathcal{C}$ include a substantial portion of the $q$-Askey scheme (Section 3.2). From the combinatorial perspective, the Hankel matrices associated to sequences obtained by specializations of $\mathcal{C}$ all have determinants that are products of squares of factorials, encompassing at least ten known such instances from the literature (Section 3.3). Specializations of $\mathcal{C}$ include a variety of well-known combinatorial sequences associated to permutations and set partitions (including perfect matchings), and also the distribution of the numbers of occurrences of several permutation patterns of length 3. As a first substantiated link between permutation patterns and moment sequences, we show that a sequence of numbers of avoiders of a pattern of length 3 is the moment sequence of a probability measure if and only if it is obtained as a specialization of $\mathcal{C}$ (Section 3.4). The continued fraction $\mathcal{C}$ also captures a host of statistics on the symmetric groups (permutations), the hyperoctahedral groups (signed permutations), and, more generally, certain types of unitary reflection groups (colored permutations) (Section 3.5). The combinatorial framework presented here leads us, in a natural way, to the definition of $k$-arrangements, permutations with $k$-colored fixed points, which include the derangements, the permutations and what had previously been called just arrangements. We present some results on the structure and enumeration of these and several conjectures (Section 3.6).

Finally, in Section 4 we give a proof for the central bijection of the paper, between permutations and Motzkin paths labeled to capture the fourteen different statistics on permutations, and explain the connections and differences between the results in this paper and those by several other authors.

\section{Definitions and the Main Theorem}

The following definitions are most naturally understood using two-row diagrams depicting permutations as bijections from the set $[n]:=\{1,2, \ldots, n\}$ to itself (see Figure 1 ). We also use one-line notation, presenting a permutation $\sigma$ as the word $\sigma(1) \sigma(2) \ldots \sigma(n)$.

The continued fraction $\mathcal{C}$ in Definition 1 has a combinatorial interpretation in terms of fourteen ele- 
mentary combinatorial statistics on permutations, defined as follows (see Figure 1 for an example):

Definition 2. Let $\sigma$ be a permutation in $\mathcal{S}_{n}$ and $[n]=\{1,2, \ldots, n\}$. For each $\sigma$ we define its

1. number of excedances as $\operatorname{exc}(\sigma):=\#\{i \in[n] \mid i<\sigma(i)\}$,

2. number of fixed points as $\operatorname{fp}(\sigma):=\#\{i \in[n] \mid i=\sigma(i)\}$,

3. number of anti-excedances as $\operatorname{aexc}(\sigma):=\#\{i \in[n] \mid i>\sigma(i)\}$,

4. number of linked excedances as $\operatorname{le}(\sigma):=\#\left\{i \in[n] \mid \sigma^{-1}(i)<i<\sigma(i)\right\}$,

5. number of linked anti-excedances as $\operatorname{lae}(\sigma):=\#\left\{i \in[n] \mid \sigma^{-1}(i)>i>\sigma(i)\right\}$.

We say that $i$ is an excedance if $i<\sigma(i)$, and likewise for the other definitions above. We also define the following statistics for each $\sigma$ :

6. The number of inversions between excedances: ie $(\sigma):=\#\{i, j \in[n] \mid i<j<\sigma(j)<\sigma(i)\}$.

7. The number of inversions between excedances where the greater excedance is linked: ile $(\sigma):=\#\left\{i, j \in[n] \mid i<j<\sigma(j)<\sigma(i)\right.$ and $\left.\sigma^{-1}(j)<j\right\}$.

8. The number of restricted non-inversions between excedances: $\operatorname{nie}(\sigma):=\#\{i, j \in[n] \mid i<j<\sigma(i)<\sigma(j)\}$.

9. The number of restricted non-inversions between excedances where the rightmost excedance is linked: $\operatorname{nile}(\sigma):=\#\left\{i, j \in[n] \mid i<j<\sigma(i)<\sigma(j)\right.$ and $\left.\sigma^{-1}(j)<j\right\}$.

10. The number of inversions between anti-excedances: $\operatorname{iae}(\sigma):=\#\{i, j \in[n] \mid j>i>\sigma(i)>\sigma(j)\}$.

11. The number of inversions between anti-excedances where the smaller anti-excedance is linked: ilae $(\sigma):=\#\left\{i, j \in[n] \mid j>i>\sigma(i)>\sigma(j)\right.$ and $\left.\sigma^{-1}(i)>i\right\}$.

12. The number of restricted non-inversions between anti-excedances: $\operatorname{niae}(\sigma):=\#\{i, j \in[n] \mid j>i>\sigma(j)>\sigma(i)\}$.

13. The number of restricted non-inversions between anti-excedances where the smaller antiexcedance is linked: nilae $(\sigma):=\#\left\{i, j \in[n] \mid j>i>\sigma(j)>\sigma(i)\right.$ and $\left.\sigma^{-1}(i)>i\right\}$.

14. The number of inversions between excedances and fixed points: $\operatorname{iefp}(\sigma):=\#\{i, j \in[n] \mid i<j=\sigma(j)<\sigma(i)\}$.

Note that $i$ is a linked excedance if and only if $i<\sigma(i)$ and the $j$ for which $\sigma(j)=i$ is also an excedance, that is, $j<\sigma(j)$. An analogous statement is true for linked anti-excedances and this is illustrated in Figure 1. Although we do not define this separately, it should be clear that exc-le counts non-linked excedances, and aexc - lae non-linked anti-excedances.

The items 6-14 in Definition 2 all refer to inversions and non-inversions, the latter with certain restrictions. A pair $(i, j)$ with $i<j$ forms an inversion if $\sigma(i)>\sigma(j)$ and a non-inversion if $\sigma(i)<\sigma(j)$. A non-inversion is restricted if we additionally require $\sigma(i)>j$ when dealing with excedances or $\sigma(j)<i$ in the case of anti-excedances. Restricted non-inversions are the natural counterparts to inversions when working within the class of excedances (or anti-excedances), as seen by comparing items 6 to 8 (resp. 10 to 14) in Definition 2 . 

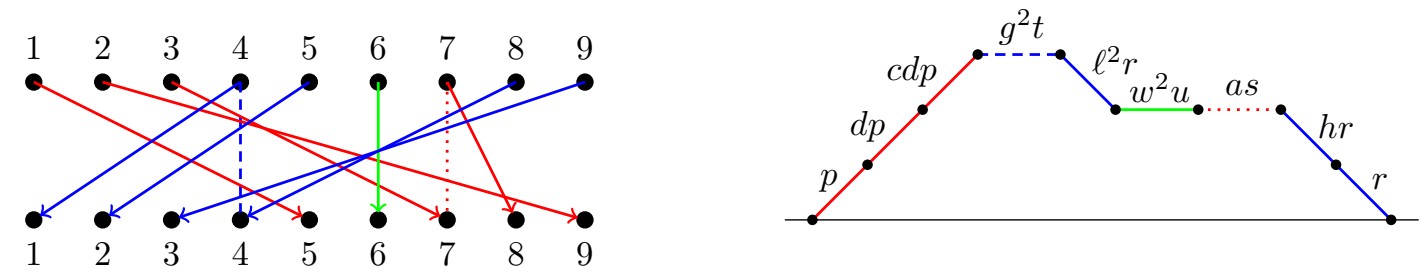

Figure 1: The permutation 597126843 and the corresponding labeled Motzkin path. Excedances are red with dotted red line indicating linked excedance, anti-excedances blue with dashed blue line indicating linked anti-excedance, fixed point green. Note that the parameters not present in the labels in this example simply have exponent 0 , such as as standing in for $a b^{0} s$.

The inversions are further refined based on whether the participating excedances or anti-excedances are linked. For example, ile $(\sigma)$ counts the number of inversions between excedances in which the greater excedance is linked. In the permutation 597126843 of Figure 1, the inversion $(2,7)$ is included in this statistic, as $\sigma(2)=9>8=\sigma(7)$ and the excedance 7 is linked. As another example with the same permutation, niae $(\sigma)$ is the number of non-inversions between anti-excedances, such as $(4,8)$, where $\sigma(4)=1<4=\sigma(8)$. Note that this non-inversion also contributes to nilae since the smaller anti-excedance 4 is linked. Finally, iefp is the sum over all fixed points $j$ of the number of excedances $i$ preceding $j$ that are greater than $j$ and thus form an inversion with $j$. In our running example the only fixed point, 6 , has two such excedances preceding it, namely 2 and 3.

The continued fraction $\mathcal{C}$ in Definition 1 gives, in the sense of formal power series, the generating function for all the statistics above. The following is the central theorem of this paper:

\section{Theorem 1.}

$$
\mathcal{C}(z)=\sum_{n \geq 0} \sum_{\sigma \in \mathcal{S}_{n}} \begin{aligned}
& a^{\text {ile }(\sigma)} b^{\text {nile }(\sigma)} c^{\text {ie }(\sigma)-\operatorname{ile}(\sigma)} d^{\text {nie }(\sigma)-\text { nile }(\sigma)} f^{\text {ilae }(\sigma)} g^{\text {nilae }(\sigma)} h^{\text {iae }(\sigma)-\text { ilae }(\sigma)} \ell^{\text {niae }(\sigma)-\text { nilae }(\sigma)} \\
& \times p^{\operatorname{exc}(\sigma)-\operatorname{le}(\sigma)} r^{\operatorname{aexc}(\sigma)-\operatorname{lae}(\sigma)} s^{\operatorname{le}(\sigma)} t^{\operatorname{lae}(\sigma)} u^{\mathrm{fp}(\sigma)} w^{\text {iefp }(\sigma)} z^{n} .
\end{aligned}
$$

Theorem 1 follows from Flajolet's correspondence [Fla80, Section 1.1] between continued fractions and labeled Motzkin paths, together with the following definition and theorem.

Recall that a Motzkin path is a polygonal path in $\mathbb{R}^{2}$, consisting of upsteps $(1,1)$, level steps $(1,0)$ and downsteps $(1,-1)$, starting at the origin and ending on the $x$-axis without ever going below it (see Figure 1).

Definition 3. Let $\mathcal{M}_{n}:=\mathcal{M}_{n}(a, b, c, d, f, g, h, \ell, p, r, s, t, u, w)$ be the set of Motzkin paths of length $n$ labeled as follows, where $I_{k}=\{0,1, \ldots, k-1\}$ :

- Upsteps from height $k-1$ to height $k \geq 1$ have labels in $\left\{p c^{i} d^{k-1-i} \mid i \in I_{k}\right\}$.

- Downsteps from height $k$ to height $k-1$ have labels in $\left\{r h^{i} \ell^{k-1-i} \mid i \in I_{k}\right\}$.

- Level steps at height $k$ have labels in

$$
\left\{u \cdot w^{i} \mid i \in I_{k}\right\} \cup\left\{s a^{i} b^{k-1-i} \mid i \in I_{k}\right\} \cup\left\{t f^{i} g^{k-1-i} \mid i \in I_{k}\right\} .
$$

The weight of a path $M \in \mathcal{M}_{n}$, denoted wt $(M)$, is the product of its labels.

The following theorem will be proved in Section 4 . 
Theorem 2. There exists a bijection $\eta: \mathcal{S}_{n} \rightarrow \mathcal{M}_{n}$ with the property that if $M=\eta(\sigma)$ then

$$
\begin{aligned}
\operatorname{wt}(M)= & a^{\text {ile }(\sigma)} b^{\text {nile }(\sigma)} c^{\text {ie }(\sigma)-\text { ile }(\sigma)} d^{\text {nie }(\sigma)-\text { nile }(\sigma)} f^{\text {ilae }(\sigma)} g^{\text {nilae }(\sigma)} h^{\text {iae }(\sigma)-\text { ilae }(\sigma)} \ell^{\text {niae }(\sigma)-\text { nilae }(\sigma)} \\
& \times p^{\operatorname{exc}(\sigma)-\operatorname{le}(\sigma)} r^{\text {aexc }(\sigma)-\operatorname{lae}(\sigma)} s^{\operatorname{le}(\sigma)} t^{\operatorname{lae}(\sigma)} u^{\mathrm{fp}(\sigma)} w^{\text {iefp }(\sigma)} .
\end{aligned}
$$

The fourteen permutation statistics listed above are basic building blocks from which a number of classical combinatorial statistics on permutations and set partitions can be constructed. Thus, by specializing the values of its parameters, the continued fraction $\mathcal{C}=\mathcal{C}_{a, b, c, d, f, g, h, \ell, p, r, s, t, u, w}(z)$ enumerates a variety of familiar objects, examples of which are listed in Table 1. Further specializations appear in various results in Sections 3.4-3.6.

\section{Corollaries and Extensions}

The central result of this paper, Theorem 1, gives a combinatorial interpretation for the continued fraction $\mathcal{C}$ in terms of fourteen elementary statistics on the symmetric groups. This unifying lens gives rise to a variety of corollaries and extensions, described at present.

\subsection{Permutation Statistics as Moment Sequences}

Consider the sequence

$$
m_{0}=1, \quad m_{n}=\left[z^{n}\right] \mathcal{C}_{a, b, c, d, f, g, h, \ell, p, r, s, t, u, w}(z) \quad(n \in \mathbb{N}),
$$

with the corresponding $\alpha_{n}, \beta_{n}$ as in (3) and the parameters $a$ through $w$ real numbers.

From the general theory of moment problems and continued fractions (e.g. [ST43]), $\left(m_{n}\right)$ is the sequence of moments of some probability measure $\mu$ on the real line if and only if $\beta_{n} \geq 0$ for all $n \in \mathbb{N}$. If $\beta_{n}>0$ for all $n<n_{0}$ and $\beta_{n_{0}}=0$, the measure $\mu$ is unique (up to equivalence) and is supported on a set of $n_{0}$ elements. If $\beta_{n}$ is strictly positive for all $n$, there may be multiple non-equivalent measures, supported on infinite sets, whose moments are given by (5).

A sufficient condition for the determinacy of the Hamburger moment problem is Carleman's condition (e.g. [ST43]), namely, $\sum_{n=1}^{\infty} \beta_{n}^{-1 / 2}=\infty$. We therefore immediately obtain the following.

Corollary 1. For $a, b, c, d, f, g, h, \ell, p, r, s, t, u, w \in \mathbb{R}$ with $p r>0$ and $c, d, h, \ell$ satisfying

$$
c=-d \quad \text { or } h=-\ell \text { or }(c>-d \text { and } h>-\ell) \text { or }(c<-d \text { and } h<-\ell) \text {, }
$$

the sequence $\left(m_{n}\right)$ in $(5)$ is the moment sequence of some probability measure on the real line. If $p=0$ or $r=0$, the measure is unique and consists of one atom; if $c=-d$ or $h=-\ell$, the measure is unique and is formed of two atoms. More generally, when $\max (|c|,|d|) \cdot \max (|h|,|\ell|) \leq 1$, the measure is unique.

In other words, the continued fraction $\mathcal{C}$ encodes moments of a very general class of probability measures on the real line whose moments have combinatorial interpretations in terms of permutations and set partitions. Table 1 lists examples of combinatorial sequences, obtained as specializations of (5), which are moment sequences of familiar measures. These include several fundamental laws in classical and noncommutative probability, such as various noncommutative central limits. Table 2 provides further examples, associated with sixteen named orthogonal polynomial sequences belonging to the $q$-Askey scheme, discussed in more detail in the following section. 
That $\mathcal{C}$ encodes such an abundance of well-studied probability measures is somewhat surprising. What is more, several of the measures in Table 1 share a curious property: as 'noncommutative analogues' of the Poisson and Gaussian laws, they are in some (noncommutative, combinatorial) sense stable.

Permutations and set partitions feature strongly in the basic constructions of noncommutative probability (see e.g. the monograph [NS06] for a combinatorial perspective on the free probability theory), but whether combinatorial statistics on permutations and set partitions also carry probabilistic meaning is generally less clear. For instance, the passage from perfect matchings to noncrossing perfect matchings is, from a probabilistic perspective, the difference between the classical Central Limit Theorem (CLT) to the free CLT (e.g. [NS06]). Moreover, perfect matchings refined for the number of crossings or those additionally refined for the number of nestings correspond, respectively and in an analogous manner, to the CLT governing a mixture of commuting/anti-commuting elements [Spe92] or the CLT describing a mixture of elements that commute with respect to a real-valued commutation coefficient [Bli12].

Overall, the existence of a relatively simple unifying combinatorial framework that encodes a broad swath of important probability laws is intriguing. It begs the question of whether the measures belonging to the family $\mathcal{C}$ can be given a similarly consistent probabilistic interpretation, potentially through the lens of noncommutative probability.

\subsection{A scheme of orthogonal polynomials}

Closely related to continued fractions are sequences of polynomials that are orthogonal with respect to some linear functional. Recall that any measure $\mu$ on the real line with finite moments, $m_{n}=$ $\int_{\mathbb{R}} x^{n} d \mu(x)<\infty$ for all $n \in \mathbb{N}$, can be associated with a continued fraction. In particular, from the combinatorial point of view, it is natural to consider the formal power series generating function $\sum_{n \geq 0} m_{n} z^{n}$, which has a continued fraction expansion of the form (2), with coefficients $\alpha_{n} \in \mathbb{R}$ and $\beta_{n} \geq 0(n \in \mathbb{N})$ uniquely determined by $\mu$. In turn, the polynomials defined by

$$
P_{0}(x)=1, \quad P_{1}(x)=x-\alpha_{0}, \quad P_{n+1}(x)=\left(x-\alpha_{n}\right) P_{n}(x)-\beta_{n} P_{n-1}(x)
$$

form an orthogonal basis of $L^{2}(\mu)$, the Hilbert space of real or complex-valued functions that are square-integrable with respect to $\mu$. The practical importance of a convenient orthogonal basis cannot be overstated and, indeed, orthogonal polynomials appear in many areas of pure and applied mathematics, especially in analysis, mathematical physics, and numerical methods. The 'classical' families, such as the Hermite, Laguerre, or Charlier (associated, respectively, with the Gaussian, Exponential, and Poisson measures), and their various $q$-analogues are brought together in the $q$-Askey scheme [KLS10].

Moments of the measures associated with hypergeometric orthogonal polynomials have been extensively studied in the combinatorial literature. For instance, while the moments of the orthogonalizing measures for the Hermite, Laguerre, and Charlier polynomials count, respectively, perfect matchings, permutations, and set partitions (see Table 1), their $q$-analogues correspond to combinatorial refinements of these structures (see e.g. [ISV87, dMSW95, SS94, SS96, KSZ11]). The aforementioned families can all be expressed as specializations of the Askey-Wilson polynomials, a four-parameter family of $q$-hypergeometric orthogonal polynomials that, along with the $q$-Racah family, is at the top of $q$-Askey hierarchy. This line of combinatorial inquiry culminated in the work of Corteel and Williams [CW11, CSSW12], who, in the context of studying the asymmetric exclusion process (ASEP), gave a combinatorial interpretation for the Askey-Wilson moments.

Namely, let $Z_{\ell}$ be the partition function associated with the so-called staircase tableaux of size $\ell$ (that is, $Z_{\ell}=\sum_{T} \operatorname{wt}(T)$, where the sum is taken over all such tableaux and the weight is the 
product of labels assigned to each tableau). Then, the $n$th moment of the Askey-Wilson polynomials in four parameters, $\alpha, \beta, \gamma, \delta$, and one ' $q$ ' is given as [CW11, CSSW12]

$$
m_{n}^{(\alpha, \beta, \gamma, \delta, q)}=\frac{(a b c d ; q)_{\infty}}{(q, a b, a c, a d, b c, b d, c d ; q)_{\infty}} \sum_{\ell=0}^{n}(-1)^{n-\ell}\left(\begin{array}{c}
n \\
\ell
\end{array}\right)\left(\frac{1-q}{2}\right)^{\ell} \frac{Z_{\ell}}{\Pi_{i=0}^{\ell-1}\left(\alpha \beta-\gamma \delta q^{i}\right)} .
$$

While the formula (8) is certainly very interesting, it takes some effort, especially when seeking bijective proofs, to specialize it to the considerably simpler combinatorial interpretations of known special cases of this family. (For this, see [CSSW12], which also contains a version of (8) without an alternating sum.)

We instead show that a substantial portion of the $q$-Askey scheme can be given a transparent combinatorial meaning in terms of the fourteen permutation statistics of Definition 2. Indeed, consider the polynomial sequence $\left(P_{n}\right)$ defined in $(7)$, with the coefficients $\left(\alpha_{n}\right)$ and $\left(\beta_{n}\right)$ as in (3), orthogonal with respect to the family of measures discussed in the preceding section. It is easy to verify that $\left(P_{n}\right)$ includes the sixteen named orthogonal polynomial sequences listed in Table 2 . This interpretation straightforwardly recovers previously studied special cases in this scheme, such as [ISV87, dMSW95, SS94, SS96, KSZ11], generalizing and unifying these into a consistent combinatorial framework.

The idea that a broader subset of the $q$-Askey scheme may be interpretable in terms of permutation statistics is due to Simion and Stanton (see Section 10 of [SS96]). At present, due to a difference in the treatment of fixed points (see Section 4.4), we are able to substantially enlarge this class of examples. It remains to be seen whether this combinatorial framework can be extended to yield a more complete elementary interpretation of the $q$-Askey scheme and related entities. (See, for example, the remark following Corollary 6 in Section 3.4 concerning the appearance in $\mathcal{C}$ of familiar linearization coefficients.) For now, the further extensions beyond the setting of the symmetric groups, described in Sections 3.5 and 3.6, and the striking connection to permutation patterns (Section 3.4) provide a new perspective on these well-studied families of orthogonal polynomials.

\subsection{The Hankel transform}

In enumerative combinatorics, the passage from a given integer sequence $\left(a_{n}\right)$ to the sequence of determinants of the Hankel matrices $\left(a_{i+j}\right)_{0 \leq i, j \leq n}$ is referred to as the Hankel transform. Radoux was among the first to undertake a systematic study of the Hankel transforms of familiar integer sequences [Rad79, Rad90, Rad91, Rad92, Rad00, Rad02]. For instance, consider the so-called exponential polynomials $\left(e_{n}\right)$,

$$
e_{0}(x)=1, \quad e_{n}(x)=\sum_{\pi} x^{|\pi|} \quad(n \in \mathbb{N}),
$$

where the sum runs over all set partitions of $[n]$ and $|\pi|$ denotes the number of blocks of a partition $\pi$. Clearly, $e_{n}(1)$ is the $n$th Bell number, while the coefficients of $e_{n}(x)$ are the Stirling numbers of the second kind. In [Rad79], Radoux showed that

$$
\operatorname{det}\left(e_{i+j}(x)\right)_{0 \leq i, j \leq n}=x^{\left(\begin{array}{c}
n+1 \\
2
\end{array}\right)} \prod_{k=1}^{n} k !
$$

(For a combinatorial proof of (10), see [Ehr00], where it also shown that the identity continues to hold when the exponential polynomials are replaced by those enumerating set partitions with block sizes of at least, at most, or exactly equal to 2.) Radoux's result is at the heart of two 
combinatorial phenomena. First, the Hankel transforms of many of the 'classical' sequences, such as the Euler numbers, Catalan numbers, numbers of involutions, and Hermite polynomials, have similarly pleasing forms. Second, many combinatorial sequences share the same Hankel transform. For example, letting $D_{n}$ denote the number of derangements (that is, permutations without fixed points) on $n$ letters and setting $D_{0}:=1$, one can show $[\operatorname{Rad} 79$, Ehr00] that

$$
\operatorname{det}\left(D_{i+j}\right)_{0 \leq i, j \leq n}=\prod_{i=1}^{n}(i !)^{2} \quad \text { for all } n \in \mathbb{N} .
$$

In addition, [OEI] lists ten other known integer sequences (viz. A000023, A000142, A000522, A003701, A010842, A010843, A051295, A052186, A053486, A053487, in addition to the derangements A000166) that share the Hankel transform given above. The emergence of a unifying theme was reinforced in subsequent work, under different perspectives (e.g. [Jun03, Wim00]).

It turns out that many of the examples studied in [Rad92, Jun03, Wim00, Ehr00] as well as all of the aforementioned sequences in [OEI] are recovered from straightforward specializations of (2). In fact, a considerably more general result is true. By standard results in the theory of orthogonal polynomials, the determinants of the Hankel matrices associated with the sequence $\left(m_{n}\right)$ are given in terms of products of the diagonal terms of the corresponding continued fraction, namely

$$
\operatorname{det}\left(m_{i+j}\right)_{0 \leq i, j \leq n}=\left(\beta_{1}\right)^{n}\left(\beta_{2}\right)^{n-1} \ldots\left(\beta_{n-1}\right)^{2} \beta_{n}
$$

(The reader is referred to [Kra99, Kra05] for historical references and some example applications of (12).) We thereby immediately obtain the following.

Corollary 2. For any sequence $\left(m_{n}\right)$ satisfying (5), we have that

$$
\operatorname{det}\left(m_{i+j}\right)_{0 \leq i, j \leq n}=(p r)^{\left(\begin{array}{c}
n+1 \\
2
\end{array}\right)} \prod_{i=1}^{n}[i]_{c, d} ![i]_{h, \ell} !,
$$

where $[i]_{c, d} !:=[i]_{c, d}[i-1]_{c, d} \ldots[1]_{c, d}$.

In other words, the structure of the Hankel transform as a product of $q$-factorials is broadly shared among integer sequences and generating functions associated with permutations and set partitions. In particular, the above corollary encompasses the combinatorial objects given in Tables 1 and 2 , as well as further specializations discussed in the next three sections. Overall, the observed similarity among Hankel transforms of familiar sequences rightfully points to a combinatorial phenomenon, the root of which has perhaps mostly to do with the preponderance of combinatorial questions that can be expressed in terms of natural statistics on permutations and set partitions.

\subsection{Permutation Patterns}

The continued fraction $\mathcal{C}$ unifies and refines several major enumerative results on permutation patterns.

Concretely, a (classical) permutation pattern of length $\ell$ is a permutation on $\ell$ letters. A permutation $\sigma \in \mathcal{S}_{n}$ contains an occurrence of the pattern $\pi$ if it includes $\pi$ as a subpermutation, that is, if there is an $\ell$-tuple $i_{i}<i_{2}<\cdots<i_{\ell}$ such that $\sigma\left(i_{j}\right)<\sigma\left(i_{k}\right)$ if and only if $\pi(j)<\pi(k)$. Moreover, $\pi$ is a consecutive pattern if its occurrences are constrained to segments $\sigma_{k} \sigma_{k+1} \ldots \sigma_{k+\ell-1}$ of $\sigma$. A vincular pattern is a pattern partitioned by dashes into blocks whose elements form consecutive patterns, so that in an occurrence of such a pattern, the letters corresponding to a block of consecutive 
letters must be consecutive in the permutation. (For example, representing elements of $\mathcal{S}_{n}$ as words on $[n]$ with each letter appearing exactly once, the subword 624 in 356214 is an occurrence of the vincular pattern 31-2; in contrast, 524 is not since 5 and 2 are not adjacent in 356214 , but 524 is an occurrence of the classical pattern 213). A permutation avoids a given pattern if it contains no occurrences of it.

Sporadic, sometimes implicit, results on permutation patterns have appeared for over a century (perhaps first in the work of MacMahon [Mac04] early last century). In the 1960s Knuth [Knu98] pointed out their connection to sorting devices in theoretical computer science, which has seen much work since then, and in 1985 appeared the seminal paper of Simion and Schmidt [SS85] who enumerated the sets of permutations avoiding any given set of patterns of length 3 . In the last two decades this has been an active area of research, with hundreds of papers published, and several new directions arising, involving asymptotics and order theoretic properties and topology of the set of all finite permutations partially ordered by pattern containment. A fairly recent survey is [Kit11], which, however, does not cover the more recent developments on the order theoretic and topological aspects.

The number of elements of $\mathcal{S}_{n}$ that avoid any single classical pattern of length 3 is the $n$th Catalan number. The numbers of permutations avoiding one of the vincular patterns 1-32 or 1-23 (and their symmetric equivalents, such as 23-1 and 32-1) are the Bell numbers [Cla01], while avoiders of the vincular pattern 2-31 (which belongs to the only other symmetry class of vincular patterns of length 3 with one dash) are enumerated by the Catalan numbers (which is explicit in [Cla01] and implicit in [CSZ97, Thm. 10], as the statistic Res there equals the number of occurrences of 2-31).

Both the Catalan and Bell numbers arise as special cases of the continued fraction $\mathcal{C}$, which can be seen by comparing $\mathcal{C}$ to the continued fractions given in Proposition 5 and Theorem 2 in [Fla80]. (In fact, it is also possible to prove this, and more - namely, including refinements involving the number of blocks in set partitions and, respectively, the number of descents in permutations counted by the Catalan numbers - via a detailed analysis of the action of the bijection $\phi$ in Lemma 1 below.) Furthermore, the permutations avoiding the consecutive pattern 123 are also enumerated by $\mathcal{C}$ (see Corollary 4 below), while the numbers of those avoiding the consecutive pattern 132 are not a moment sequence (as evidenced by the computation of a $6 \times 6$ Hankel determinant) and, indeed, cannot be recovered from $\mathcal{C}$ (for this would require $\beta_{1}=1$ and $\beta_{5}<0$, which is inconsistent with (3)). These are the only symmetry classes of patterns of length 3 . We therefore obtain the following corollary to Theorem 1.

Corollary 3. Let $\pi$ be a pattern of length 3 and denote by $x_{n}$ the number of elements of $\mathcal{S}_{n}$ that avoid $\pi$. Then $\left(x_{n}\right)$ is a sequence of moments of some (positive Borel) measure on the real line if and only if the ordinary generating function of $\left(x_{n}\right)$ is a special case of $\mathcal{C}$.

While the enumerative results for the patterns encompassed by Corollary 3 are well known, the result itself is more than a sum of its parts. Specifically, the study of permutation patterns varies greatly in difficulty depending on the choice of the pattern, ranging from fine-grained results for consecutive patterns [EN03] to speculations on ever computing the number of avoiders of the classical pattern 1324 [Egg15]. The latter is perhaps the most active front in the search for enumeration of single patterns, both exact and asymptotic, and only incremental progress has been made during the last decade in the enumeration of avoiders of this pattern. Conway, Guttmann and Zinn [CGZJ18] pushed the exact enumeration of 1324 -avoiders up to $n=50$ with a powerful algorithm, and used that to give an estimate for the asymptotics of this sequence. Based on this data, Elvey-Price has conjectured [EP18] that the numbers of 1324-avoiders form a moment sequence. Improving bounds have also been emerging for this pattern [BBEP17], but they are still very wide, and no general methods seem to be on the horizon for exact enumeration of 1324 avoiders, let alone of more intricate 
patterns. However, Corollary 3 gives hope that there are nevertheless general threads connecting these seemingly disparate problems and that general results may yet be achievable in this setting.

Corollary 3 does in fact extend to the numbers of occurrences of two of the patterns mentioned above. In [EN03, Thm. 4.1], Elizalde and Noy gave an explicit (but complicated) expression for the exponential generating function for the number of occurrences of the consecutive pattern 123 in permutations, and in [Eli17, Eq. (5)] Elizalde gave a continued fraction for the corresponding ordinary generating function. Of course, this distribution is equal to the distribution of the number of occurrences of the consecutive pattern 321 , denoted occ $_{321}$. As a corollary of our main theorem, the continued fraction $\mathcal{C}$ refines this result further, giving the joint distribution of $\operatorname{occ}_{321}$ with the number of descents (places $i$ such that $\sigma_{i}>\sigma_{i+1}$ ). First we recall a bijection that we will use several times in what follows.

Lemma 1 ([Ste94, Thm. 51]). Given $\sigma=a_{1} a_{2} \ldots a_{n} \in \mathcal{S}_{n}$ define $\phi: \mathcal{S}_{n} \rightarrow \mathcal{S}_{n}$ by declaring $a_{0}=0$ and setting $\phi(\sigma)=b_{1} b_{2} \ldots b_{n}$, where:

1. if $a_{i}>a_{j}$ for some $j>i$ then $b_{a_{i+1}}=a_{i}$, that is, $a_{i}$ is then in place $a_{i+1}$ in $\phi(\sigma)$,

2. if $a_{i}<a_{j}$ for all $j>i$, find the rightmost letter smaller than $a_{i}$. If this letter is $a_{k}$ then $b_{a_{k+1}}=a_{i}$, that is, then $a_{i}$ is in place $a_{k+1}$ in $\phi(\sigma)$.

Then $\phi$ is a bijection.

As an example, $\phi(264135)=413652$. As it turns out, the bijection $\phi$ is a variation on the transformation fondamentale of Foata and Schützenberger [FS70]). Note that $\phi$ takes descents 'verbatim' to excedances, in the sense that if $j$ immediately precedes $i$ in $\sigma$ and $j>i$, so that $\ldots j i \ldots$ forms a descent in $\sigma$, then $\tau=\phi(\sigma)$ has $\tau(i)=j$, so that $i$ is an excedance in $\tau$.

Corollary 4. Setting $s=q x, p=x$, and all other parameters to 1, we obtain

$$
\mathcal{C}(z)=\sum_{n \geq 0} \sum_{\sigma \in \mathcal{S}_{n}} x^{\operatorname{des}(\sigma)} q^{\text {occ } 321}(\sigma) z^{n},
$$

where occ 321 is the number of occurrences of the consecutive pattern 321 and des denotes the number of descents.

Proof. An occurrence of the pattern 321 is a double descent, defined as an $i$ such that $\sigma_{i-1}>\sigma_{i}>$ $\sigma_{i+1}$. Suppose a permutation $\sigma$ has a double descent involving the letters $i, j, k$, appearing in that order, so $i>j>k$. Applying $\phi$ to $\sigma$ will result in a permutation $\tau$ with $\tau(k)=j$ and $\tau(j)=i$, which is precisely a linked excedance. Since the parameter $s$ carries linked excedances in $\mathcal{C}$, each linked excedance contributes a factor of both $x$ and $q$ here, whereas the non-linked excedances, carried by $p$ in $\mathcal{C}$, contribute only an $x$.

This connection between the occurrences of the pattern 321 and the continued fraction $\mathcal{C}$ is fundamental. Indeed, each occurrence of the consecutive pattern 321 corresponds, in the simple manner described above, to a linked excedance, which is one of the basic statistics carried by $\mathcal{C}$. In an analogous manner, descents correspond to arbitrary excedances. ${ }^{1}$

\footnotetext{
${ }^{1}$ The double descents appearing in the proof of Corollary 4 are related to the 'double falls', whose distribution in the form of a continued fraction was given by Flajolet [Fla80, Theorem 3A]. These are defined as our double descents, except that Flajolet's double falls count also a single descent at the end of a permutation, since he appends a 0 to a permutation when computing these.
} 
It is further worth noting that the bijection $\phi$, in addition to pairing linked excedances with double descents, pairs linked anti-excedances with double ascents. Moreover, it is straightforward to show that if $\sigma$ has no linked excedances, no linked anti-excedances and no fixed points, then $\phi(\sigma)$ has no double descents or double ascents and also begins and ends with a descent (and thus must have even length). In other words, $\phi(\sigma)$ is then an alternating permutation and these are known to be counted by the (even) Euler numbers when $n$ is even. This bijection thus proves the following, since $s, t$ and $u$ carry, respectively, linked excedances, linked anti-excedances and fixed points:

Corollary 5. The number of permutations in $\mathcal{S}_{n}$ with no fixed points, no linked excedances and no linked anti-excedances is the Euler number $E_{n}$ if $n$ is even, and 0 if $n$ is odd. The generating function for the even Euler numbers is therefore obtained from $\mathcal{C}$ by setting $s=t=u=0$ and all other parameters to 1 .

Remark 1. If we also set $h=0$ in Corollary 5 the corresponding alternating permutations correspond exactly to perfect matchings. Namely, $h$ carries inversions among non-linked anti-excedances (and thus among all anti-excedances when there are no linked anti-excedances). But the antiexcedances of $\sigma$ are the 'descent bottoms' of $\phi(\sigma)$, which are the letters in even-numbered places in $\sigma$. An example is the alternating permutation 71428365 , and this is the unique such permutation corresponding to the perfect matching $(1,7)-(2,4)-(3,8)-(5,6)$.

The continued fraction $\mathcal{C}$ also encodes the number of occurrences of the vincular pattern 2-31. This distribution was already determined by Claesson and Mansour [CM02, Thm. 22] who gave a continued fraction for the joint distribution with the number of descents, the number of ascents and of the pattern 31-2. At present, we recover from $\mathcal{C}$ the following bivariate generating function, the proof following by a simple manipulation of the continued fraction in Theorem 10 in [CSZ97], noting that the statistic Res in that paper equals number of occurrences of 2-31.

Corollary 6. With $b=d=g=\ell=q, \quad s=x q, \quad p=u=x$, and all other parameters set to 1 , we have

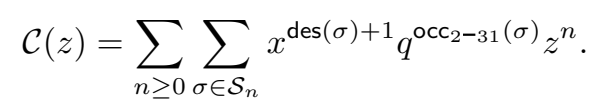

There is a more illuminating way of proving Corollary 6 , which shows how this distribution arises naturally from $\mathcal{C}$. Namely, it was shown in [SW07b, Thm. 24] that the distribution of (des +1, occ $\left._{2-31}\right)$ equals that of (wex, cross) on $\mathcal{S}_{n}$, where wex $(\sigma)$ is the number of weak excedances in $\sigma$, that is, $i$ such that $\sigma(i) \geq i$, which is equal to $\operatorname{exc}(\sigma)+\mathrm{fp}(\sigma)$. The number of crossings $\operatorname{cross}(\sigma)$ was defined in [Cor07, Def. 1] (see [SW07b, Def. 13]), and a straightforward comparison shows that it equals $\operatorname{nie}(\sigma)+\operatorname{niae}(\sigma)+\mathrm{le}(\sigma)$, leading to the parameter assignments in the corollary. It is interesting to note that this distribution was in [KSZ11, Thm. 5] shown to coincide with the linearization coefficients of the q-Laguerre polynomials.

Overall, the continued fraction $\mathcal{C}$ encodes a variety of combinatorial objects associated to permutation patterns and permutation statistics. As such, it allows us to recover and refine several key enumerative results in this area, while providing a unifying lens through which these can be viewed. In particular, the fact that $\mathcal{C}$ encodes all avoiders of patterns of length 3 (classical, vincular and consecutive) that are moment sequences gives us hope that the seemingly specialized questions in permutation patterns may be more effectively approached as instances of a more general problem.

\subsection{Generalization to the signed and colored permutations}

The continued fraction $\mathcal{C}$ enumerates elements of the symmetric groups according to the fourteen combinatorial statistics described in Definition 2. By rather simple substitutions of parameters, 
however, we obtain the distribution of analogous statistics on $k$-colored permutations, which are permutations on $n$ letters, where each letter is assigned an integer (color) from $\{0,1, \ldots, k-1\}$. The case $k=2$ specializes to the hyperoctahedral group of signed permutations. More generally, the colors of the letters factor into the definitions of descents and various other classical permutation statistics, as first defined in [Ste94] for general $k$ and outlined at present.

Definition 4. The set of $k$-colored permutations of $[n]$, denoted $\mathcal{S}_{n}^{k}$, consists of all pairs $(\sigma, c)$ where $\sigma=a_{1} a_{2} \ldots a_{n}$ is a permutation of $[n]$ and $c=c_{1} c_{2} \ldots c_{n}$ where each $c_{i}$ is an element of $\{0,1, \ldots, k-1\}$. We refer to $c_{i}$ as the color of the letter $a_{i}$. We define a total order on the pairs $\left(a_{i}, c_{i}\right)$ by setting $\left(a_{i}, c_{i}\right)>\left(a_{j}, c_{j}\right)$ if $c_{i}>c_{j}$, or $c_{i}=c_{j}$ and $a_{i}>a_{j}$. Moreover, when needed for definitions of statistics, we declare $a_{n+1}=n+1$ and $c_{n+1}=0$.

We now define five statistics on $\mathcal{S}_{n}^{k}$, following [Ste94]:

Definition 5. Let $P=\left(a_{1} a_{2} \ldots a_{n}, c_{1} c_{2} \ldots c_{n}\right)$ be a $k$-colored permutation in $\mathcal{S}_{n}^{k}$ :

1. A descent in $P$ is an $i \leq n$ such that $\left(a_{i}, c_{i}\right)>\left(a_{i+1}, c_{i+1}\right)$. The number of descents in $P$ is denoted $\operatorname{des}(P)$.

2. An excedance in $P$ is an $i \leq n$ such that $a_{i}>i$, or $a_{i}=i$ and $c_{i}>0$. The number of excedances in $P$ is denoted $\operatorname{exc}(P)$.

3. An anti-excedance in $P$ is an $i \leq n$ such that $a_{i}<i$. The number of anti-excedances in $P$ is denoted $\operatorname{aexc}(P)$.

4. An inversion in $P$ is a pair $(i, j)$ such that $i<j \leq n+1$ and $\left(a_{i}, c_{i}\right)>\left(a_{j}, c_{j}\right)$. The number of inversions in $P$ is denoted $\operatorname{inv}(P)$.

5. A fixed point in $P$ is an $i \leq n$ such that $a_{i}=i$ and $c_{i}=0$. The number of fixed points in $P$ is denoted fix $(P)$.

Note that in contrast to the symmetric group $(k=1), n$ is a descent in $\sigma=(\pi, c) \in \mathcal{S}_{n}^{k}$ when $a_{n}$ has a positive color $\left(c_{n}>0\right)$, and $n$ is an excedance when $a_{n}=n$ and $c_{n}>0$. Anti-excedances, however, coincide with anti-excedances of $\pi$. Also, any $i \leq n$ forms an inversion with $(n+1)$ if and only if $c_{i}>0$. Moreover, a fixed point requires $a_{i}=i$ and $c_{i}=0$, which matches the definition of excedances including fixed points $i$ of $\pi$ with $c_{i}>0$.

The best known case of colored permutations is that of the signed permutations $(k=2)$, frequently viewed as elements of the hyperoctahedral groups. The signed permutations have been extensively studied from many different perspectives, and most statistics on the symmetric groups have been generalized to these and analogous results obtained about the distributions of such statistics.

The colored permutations can be seen as elements of certain unitary groups generated by reflections (see [She53]) that coincide with the wreath product of the symmetric group $\mathcal{S}_{n}$ with the cyclic group $\mathbb{Z}_{k}$. Some of the classical permutation statistics were generalized to these colored permutations and many of their properties elicited in [Ste94], and in several subsequent papers by other authors (see [BGM14] for some references).

We start by showing that $\mathcal{C}$ encodes the Eulerian numbers of type $\mathrm{B}$, that is, the descent statistic on the signed permutations, prior to generalizing the result to all colored permutations. We do this for excedances - which were shown in [Ste94] to be equidistributed with descents - as part of a joint distribution with anti-excedances and fixed points.

In [FS70, Thm. 4.2], Foata and Schützenberger gave an exponential generating function for the joint distribution of (exc, fix) on the symmetric group. We now give this joint distribution over the $k$-colored permutations, incorporating also anti-excedances. 
Corollary 7. By setting $s=p=k x, t=r=k y, u=(k-1) x+q$ in $\mathcal{C}$, and all other parameters to $1, \mathcal{C}$ becomes the generating function of the joint distribution of excedances and fixed points on the colored permutations $\mathcal{S}_{n}^{k}$, that is,

$$
\mathcal{C}(z)=\sum_{n \geq 0} \sum_{\sigma \in \mathcal{S}_{n}^{k}} x^{\operatorname{exc}(\sigma)} y^{\operatorname{aexc}(\sigma)} q^{\mathrm{fix}(\sigma)} z^{n} .
$$

Proof. Setting $s=p=k x$ lets $x$ carry linked excedances, since, letting $\sigma=(\pi, c)$, an excedance $i$ in $\pi$ is an excedance in $\sigma$ regardless of $c_{i}$, the color. The same is true for non-linked excedances carried by $p$, and likewise for anti-excedances, carried by $t$ and $r$. The only complication is that a fixed point $i$ in $\pi$ is a fixed point of $\sigma$ only if $c_{i}=0$, and is otherwise an excedance. This is reflected in $u$, which carries fixed points in the case of the symmetric group $(k=1)$. Since $u$ is now the sum of $q$ and $(k-1) x$, that is, $q$ for the fixed point case when $c_{i}=0$ and $(k-1) x$ for the remaining $k-1$ choices for $c_{i}$, we recover excedances at $i$.

Although we present Corollary 7 only in this simple form, it is straightforward to refine it to incorporate the obvious generalizations of linked vs. non-linked excedances and anti-excedances to colored permutations.

Setting $w=0$ in $\mathcal{C}$ gives $u \cdot w^{n}=0$ for all $n \in \mathbb{N}$, while $u \cdot w^{0}=u$. This allows us to obtain the distribution of the number of inversions on the symmetric group and, more generally, on the $k$-colored permutations, as follows:

Corollary 8. With

$$
a=c=h=r=q, \quad b=f=d=\ell=t=q^{2}, \quad g=w=0, \quad p=u=1, \quad s=2 q,
$$

the continued fraction $\mathcal{C}$ recovers the generating function for the distribution of inversions over $\mathcal{S}_{n}$ :

$$
\mathcal{C}(z)=\sum_{n \geq 0} \sum_{\pi \in \mathcal{S}_{n}} q^{\operatorname{inv}(\pi)} z^{n} .
$$

If, in addition, we replace $z$ by $z((k-1) q+1)$, then $\mathcal{C}$ gives the distribution of inversions over the $k$-colored permutations $\mathcal{S}_{n}^{k}$ for $k>1$.

Proof. In [Bia93, Equation 1.1], Biane gives a continued fraction for the distribution of the number of inversions on $\mathcal{S}_{n}$. It is a straightforward calculation to show that Biane's continued fraction coincides with our $\mathcal{C}$ with parameters set as in the statement of this proposition.

Replacing $z$ by $z \cdot((k-1) q+1)$ is equivalent to attaching to each letter in a permutation any one of $(k-1)$ different $q \mathrm{~s}$ (which we don't need to distinguish between) or nothing, the latter corresponding to the unadorned $z$ in the case of the symmetric group $(k=1)$. With $(\pi, c)=\sigma \in \mathcal{S}_{n}^{k}$, this corresponds to the fact that only one choice of $c_{i}$ (namely 0 ) for a letter $a_{i}=\pi(i)$ with $i \leq n$ contributes a non-inversion with the appended letter $a_{n+1}=n+1$ (which has color 0 ). Each of the other $(k-1)$ choices for $c_{i}$ contributes 1 to the total number of inversions for that permutation.

Moreover, in the symmetric group, the above can be further refined to a joint distribution of inversions and excedances:

Corollary 9. Setting $p=x, s=(1+x) q$, and all other parameters as in Corollary 8, yields

$$
\mathcal{C}(z)=\sum_{n \geq 0} \sum_{\pi \in \mathcal{S}_{n}} x^{\operatorname{exc}(\pi)} q^{\operatorname{inv} \pi} z^{n}
$$


Proof. In [CSZ97, Thm. 10] a continued fraction is given that captures the distribution of (exc, inv) on the symmetric group, since inv is the sum of the statistics Edif and Ine in that paper. This continued fraction, expressed in the form for our $\mathcal{C}$, and setting $p=q$ to let $q$ carry inv, has $\widetilde{\alpha}_{n}$ and $\widetilde{\beta}_{n}$ as follows:

$$
\widetilde{\alpha}_{n}=q^{n}\left(x \cdot[n]_{q}+[n+1]_{q}\right), \quad \widetilde{\beta}_{n}=x \cdot q^{2 n-1}\left([n]_{q}\right)^{2} .
$$

The result follows by a straightforward comparison to (3) with the above parameter choices.

Whether the joint distribution of excedances and inversions over $\mathcal{S}_{n}^{k}$ can be obtained from $\mathcal{C}$ with appropriate parameter settings remains to be seen. Also worth investigating is whether other EulerMahonian pairs (see e.g. [SW07a] for examples) can be obtained from $\mathcal{C}$, such as descents and the major index, which is the sum of the descents in a permutation.

\subsection{The $k$-arrangements}

The fact that the parameter $u$ in $\mathcal{C}$ carries fixed points in permutations suggests an obvious generalization. We define a $k$-arrangement of $[n]$, for any $k \in \mathbb{N}_{0}$, to be a permutation of $[n]$ each of whose fixed points is colored with any of $k$ colors, with the convention that a 0 -arrangement is a derangement of $[n]$ (a permutation with no fixed points). Letting $k=1$ recovers ordinary permutations and $k=2$ corresponds to what previously have been called simply 'arrangements' [Com74], which also coincide with Postnikov's definition of 'decorated permutations' [Pos, Def. 13.3].

Equivalently, a $k$-arrangement of $[n]$ is a choice of $k$ disjoint subsets (some possibly empty) of $[n]$ together with a derangement of the complement of their union. An example of a 3-arrangement of [7], where we denote the three available colors by $a, b, c$, is 6214573 , with the fixed points $2,4,5$ colored $c, a, a$, respectively. This could be presented as $6 c 1 a a 73$, or, more concisely, as $3 c 1 a a 42$, to present the derangement part as a derangement of $\{1,2,3,4\}$. (Note that the original values of the letters in the derangement part can be recovered from their relative order and the set of fixed points, so the two are indeed equivalent.) Formally:

Definition 6. Let $n \in \mathbb{N}$. For a permutation $\pi \in \mathcal{S}_{n}$, denote by $\operatorname{Fix}(\pi)$ the set of fixed points of $\pi$.

1. For any $k \in \mathbb{N}$, a $k$-arrangement of $[n]$ is a pair $\mathfrak{a}=(\pi, \phi)$ where $\pi$ is a permutation of $[n]$ and $\phi: \operatorname{Fix}(\pi) \rightarrow[k]$ an arbitrary map. A 0-arrangement of $[n]$ is a derangement of $[n]$.

2. For any $k \in \mathbb{N}_{0}$, the set of $k$-arrangements of $[n]$ is denoted $A_{n}^{k}$. The set $A_{0}^{k}$, for any $k \geq 0$, consists of the empty word.

In what follows, when we apply the statistics on $\mathcal{S}_{n}$ in Definition 2 to $\mathfrak{a}=(\pi, \phi) \in \mathrm{A}_{\mathrm{n}}^{\mathrm{k}}$ we are applying them to $\pi$. This yields the following generalization of Theorem 1 .

Proposition 1. Let $\mathbf{u}=u_{1}+u_{2}+\cdots+u_{k}$. Given $\mathfrak{a}=(\pi, \phi) \in \mathrm{A}_{\mathfrak{n}}^{\mathbf{k}}$ let

$$
\operatorname{col}(\mathfrak{a})=\prod_{i \in \operatorname{Fix}(\pi)} u_{\phi(i)} .
$$

Then, letting $\mathcal{C}_{\mathbf{u}}$ be the continued fraction $\mathcal{C}$ in Definition 1 with $\mathbf{u}$ substituted for $u$, we have

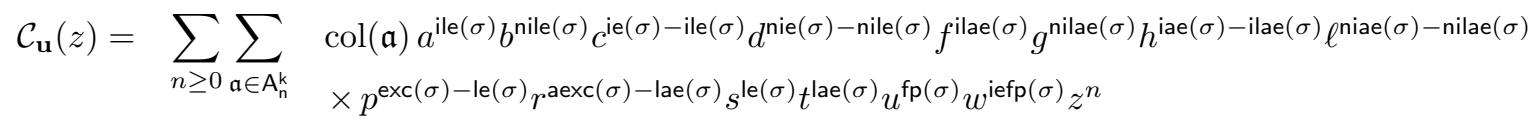


Proof. Note first that all the statistics in the exponents of parameters in (15) carry over directly to $k$-arrangements from Theorem 1 , since they are applied only to the permutation $\pi$.

Let $\mathbf{S}$ denote the summand in (4) (equivalently, the summand in (15) take away $\operatorname{col}(\mathfrak{a})$ ). Replacing $u$ in $\mathcal{C}$ by $\mathbf{u}$ means replacing $u^{\mathrm{fp}(\sigma)} \mathrm{S}$ by $\mathbf{u}^{\mathrm{fp}(\sigma)} \mathrm{S}$ in $\mathcal{C}$. That is equivalent to attaching $\mathbf{u}^{\mathrm{fp}(\sigma)}$ to each permutation $\sigma \in \mathcal{S}_{n}$ (by Theorem 1 and the underlying bijection described in Section 4). Expanding $\mathbf{u}^{\mathrm{fp}(\sigma)}$ non-commutatively turns it into $k^{\mathrm{fp}(\sigma)}$ monomials in the $u_{i}$, each of degree $\mathrm{fp}(\sigma)$. Each such monomial corresponds in a straightforward way to a coloring of the fixed points of $\sigma$, where the $i$ th fixed point gets color $u_{j}$ if $u_{j}$ is the $i$ th factor in the corresponding monomial. Conversely, each coloring of fixed points in $\sigma$ corresponds in the same simple way to one of the monomials in $\mathbf{u}^{\mathrm{fp}(\sigma)}$, where the $i$ th factor in such a monomial is the color $u_{i}$ of the $i$ th fixed point. But the colorings of fixed points in $\sigma$ are represented precisely by the monomials $\operatorname{col}(\mathfrak{a})$. Thus, replacing $u^{\mathrm{fp}(\sigma)}$ by $\mathbf{u}^{\mathrm{fp}(\sigma)}$ in $\mathcal{C}$, and hence in its expansion as in Theorem 1, corresponds to summing over all $\mathfrak{a} \in A_{n}^{k}$ with a factor of $\operatorname{col}(\mathfrak{a})$ for each of them replacing $u^{\mathrm{f} p(\sigma)}$.

Remark 2. Since replacing $u$ by $u_{1}+u_{2}+\cdots+u_{k}$ in (3) only affects the terms $\alpha_{n}$ and not $\beta_{n}$, the results in Sections 3.1 and 3.3, including Corollaries 1 and 2, are satisfied by $\mathcal{C}_{\mathbf{u}}$.

We now define two representations of $k$-arrangements, which give more insight into their structure.

Definition 7. The derangement form of an element of $A_{n}^{k}$ is a word $\mathfrak{a}$ on the alphabet

$$
\{-k,-k+1, \ldots,-1,1,2, \ldots, n-K\},
$$

where $K$ is the number of negative letters in $\mathfrak{a}$, the positive letters appear once each, and the restriction of $\mathfrak{a}$ to its positive letters is a derangement of $[n-K]$, called the derangement part of $\mathfrak{a}$. The permutation form of an element of $A_{n}^{k}$ is a word $\mathfrak{a}$ on the alphabet

$$
\{-k+1, \ldots,-1,1,2, \ldots, n-K\},
$$

where $K$ is the number of negative letters in $\mathfrak{a}$, the positive letters appear once each, and the restriction of $\mathfrak{a}$ to its positive letters is a permutation of $[n-K]$, called the permutation part of $\mathfrak{a}$.

Given a $k$-arrangement $\mathfrak{a} \in \mathrm{A}_{\mathrm{n}}^{\mathrm{k}}$, expressed as a permutation $\pi=a_{1} a_{2} \ldots a_{n}$ together with a map $\phi: \operatorname{Fix}(\pi) \rightarrow[k]$ we obtain the derangement form of $\mathfrak{a}$ by changing $a_{i}$ to $-\phi(i)$ for each fixed point $i$ of $\pi$ and then replacing the $j$-th largest positive letter left by $j$. To get the permutation form of $\mathfrak{a}$ we do the same except that we leave fixed points $i$ with $\phi(i)=k$ intact before modifying the positive letters. Each of these transformations is clearly invertible, justifying Definition 7.

As an example, the 4 -arrangement $\pi=6214573$ with $\phi(2)=4, \phi(4)=1, \phi(5)=4$ has derangement form 3-41-1-442, whose derangement part is 3142 , and permutation form 521-1463, whose permutation part is 521463 . As a $k$-arrangement for any $k>4$, however, the permutation form of $(\pi, \phi)$ equals the derangement form.

The numbers $A_{k}(n)$ of $k$-arrangements of $[n]$ satisfy a simple recursion equivalent to a simple exponential generating function, showing them to be an infinite family enumerated by successive binomial transforms:

Proposition 2. Let $A_{k}(n)$ be the number of k-arrangements of $[n]$. Then

1. $A_{k}(0)=1$ and, for $n>0, A_{k}(n)=n \cdot A_{k}(n-1)+(k-1)^{n}$.

2. The exponential generating function for $A_{k}(n)$ is $\sum_{n \geq 0} A_{k}(n) \frac{x^{n}}{n !}=e^{(k-1) x} /(1-x)$.

3. $A_{k}(n)$ equals the permanent of the $n \times n$ matrix with $k$ on the diagonal and $1 s$ elsewhere. 
4. The sequence $\left(A_{k}(n)\right)_{n \geq 0}$ is the binomial transform of the sequence $\left(A_{k-1}(n)\right)_{n \geq 0}$, that is, $A_{k}(n)=\sum_{i \geq 0}\left(\begin{array}{c}n \\ i\end{array}\right) A_{k-1}(i)$.

Proof. We will show how to generate all elements of $A_{n}^{k}$ from those of $A_{n-1}^{k}$, counting them along the way. Recall from Definition 6 that the permutation form of an element of $A_{n-1}^{k}$ is a word $\mathfrak{a}$ consisting of any $K$ negative letters from $\{-k+1,-k+2, \ldots,-1\}$, where $0 \leq K \leq n-1$ and the positive letters $\{1,2, \ldots, n-1-K\}$ once each. For any such $k$-arrangement $\mathfrak{a}$ let $M$ be its maximum letter, except $M=0$ if all letters of $\mathfrak{a}$ are negative. We can then insert a new maximum $M^{\prime}=M+1$ in any one of the $n$ places before or after a letter in $\mathfrak{a}$ to obtain words with letters from $\{-k+1,-k+2, \ldots,-1,1,2, \ldots, n-K\}$ that contain at least one positive letter. Clearly this is injective, since if two resulting words are equal they have the unique maximum letter, in the same place, and removing it gives two identical preimages. Conversely, any $k$-arrangement of $[n]$ that has some positive letters has a unique maximum letter that when removed leaves a $k$-arrangement of $[n-1]$ (possibly with only negative letters).

This thus produces, from the $k$-arrangements of $[n-1]$, all $k$-arrangements of $[n]$ that contain at least one positive letter, and thus $n$ such $k$-arrangements of $[n]$ for each $k$-arrangement of $[n-1]$, or $n \cdot A_{k}(n-1)$ in total. What remains are the $k$-arrangements of $[n]$ with only negative letters, of which there are $(k-1)^{n}$.

This recursion leads to the exponential generating function claimed, via the known exponential generating function for derangements $(k=0)$, induction and a straightforward manipulation.

That $A_{k}(n)$ equals the permanent of the $n \times n$ matrix $M_{n}^{k}$ with $k$ on the diagonal and 1 s elsewhere is easily derived from the definition of the permanent and the fact that the permanent of $M_{n}^{1}$ counts permutations of $[n]$. Namely, a $k$ appearing in the expansion of the permanent of $M_{n}^{k}$ corresponds to a fixed point in a permutation, as each $k$ comes from the diagonal of $M_{n}^{k}$, and thus counts the $k$ possible choices for coloring that fixed point.

Finally, it is well known (and easy to prove) that the binomial transform of an exponential generating function $f(x)$ is $e^{x} \cdot f(x)$ which implies $\left(A_{k}(n)\right)_{n \geq 0}$ is the binomial transform of $\left(A_{k-1}(n)\right)_{n \geq 0}$.

Remark 3. Interestingly, all the properties of Proposition 2 hold even for negative $k$, and it seems that for any $k$ the $A_{k}(n)$ eventually become positive for $n$ sufficiently large, so an obvious question is whether the $A_{k}(n)$ also enumerate some interesting combinatorial structures for $k<0$.

The definitions of descents, excedances, inversions and major index for permutations generalize in a natural way to the derangement and permutation forms of $k$-arrangements as follows.

Definition 8. Given the derangement form or permutation form $\mathfrak{a}=a_{1} a_{2} \ldots a_{n}$ of a $k$-arrangement let $s_{1} s_{2} \ldots s_{n}$ be the word consisting of the letters of $\mathfrak{a}$ sorted in weakly increasing order.

1. An excedance in $\mathfrak{a}$ is an $i$ such that $a_{i}>s_{i}$. The number of excedances in $\mathfrak{a}$ is denoted exc(a).

2. A descent in $\mathfrak{a}$ is an $i$ such that $a_{i}>a_{i+1}$. The number of descents in $\mathfrak{a}$ is denoted $\operatorname{des}(\mathfrak{a})$.

3. The major index of $\mathfrak{a}$, denoted maj(a), is the sum of the descents of $\mathfrak{a}$.

4. An inversion in $\mathfrak{a}$ is a pair $(i, j)$ such that $i<j$ and $a_{i}>a_{j}$. The number of inversions in $\mathfrak{a}$ is denoted $\operatorname{inv}(\mathfrak{a})$.

We present below some results and several conjectures for the above statistics. Which other statistics on permutations generalize in natural ways to $k$-arrangements remains to be elicited, as well as the distributions of such statistics. First we present a property of $k$-arrangements in the permutation 
form, allowing us to exploit the substantial knowledge on rearrangement classes, which are sets of permutations of multisets.

Definition 9. Let $\mathfrak{a}$ be a word of length $n$ on some alphabet, and $M(\mathfrak{a})$ the multiset of all letters in $\mathfrak{a}$. The rearrangement class of $\mathfrak{a}$ is the set of words of length $n$ whose multiset of letters is $M(\mathfrak{a})$.

As an example, the rearrangement class of 0110 consists of $\{0011,0101,0110,1001,1010,1100\}$.

Proposition 3. The set of elements of $\mathrm{A}_{\mathrm{n}}^{\mathrm{k}}$ in permutation form, for any $n$ and $k$, is a union of rearrangement classes.

Proof. The permutation form of a $k$-arrangement $\mathfrak{a} \in \mathrm{A}_{\mathrm{n}}^{\mathrm{k}}$ consists of any multiset of size $s \leq n$ of negative numbers in $\{-1,-2, \ldots,-k+1\}$ and each of the positive numbers in $[n-s]$, once each. Every rearrangement of $\mathfrak{a}$ consists of the same multiset of negative numbers and the same positive numbers and thus is the permutation form of some element of $A_{n}^{k}$.

It was shown already by MacMahon [Mac04] early last century that exc and des (as defined above) are equidistributed on the rearrangement class of any word, and that the same applies to inv and maj. Although rearrangement classes are typically defined on words containing all the letters in $[k]$ for some $k$, statistics on them often depend only on the relative sizes of letters. Thus, thanks to Proposition 3, we have the following result.

Proposition 4. Suppose $s$ and $s^{\prime}$ are two statistics on words of integers that depend only on the relative sizes of those integers, and suppose $s$ and $s^{\prime}$ are equidistributed on the rearrangement class of any word. Then $s$ and $s^{\prime}$ are equidistributed on $A_{n}^{k}$ in permutation form for any $n$ and $k$.

Since the definitions of exc, des, inv and maj only depend on relative sizes of letters in $k$-arrangements, we have the following result.

Proposition 5. The statistics exc and des, and, respectively, inv and maj, are equidistributed on elements of $\mathrm{A}_{\mathrm{n}}^{\mathrm{k}}$ in their permutation form for any $n$ and $k$.

Conjecture $1 .^{2}$ The statistic des has the same distribution on elements of $\mathrm{A}_{\mathrm{n}}^{\mathrm{k}}$ in their derangement form and permutation form for any $n$ and $k$.

In the remainder of this subsection, all permutation patterns are taken to be classical.

It is well known that the number of permutations avoiding any single permutation pattern of length 3 is the $n$-th Catalan number $C(n)=\frac{1}{n+1}\left(\begin{array}{c}2 n \\ n\end{array}\right)$. An analogous statement is true of the 2arrangements in their permutation form. The definition of occurrence of a pattern extends trivially to $k$-arrangements in their permutation (or derangement) form, but an occurrence of such a pattern must involve different letters, and can thus not have more than one copy of any of the negative letters. (There are, however, generalizations of patterns that allow repeated letters in occurrences, and studying these for the $k$-arrangements would be natural. For information on such patterns see [Kit07] and [Kit11, Section 1.5]).

Proposition 6. The number of 2-arrangements of $[n]$ whose permutation form avoids any single permutation pattern of length 3 is the Catalan number $C(n+1)$. Moreover, the number of such 2-arrangements with $k$ negative numbers in their permutation form is the ballot number $\frac{k+1}{n+1}\left(\begin{array}{c}2 n-k \\ n\end{array}\right)$.

\footnotetext{
${ }^{2}$ In response to the preprint of this article posted on arxiv.org, Fu, Han and Lin [FHL20] have recently proved Conjectures 1 through 4 in this section.
} 
Proof. We first exhibit a bijection between 312-avoiding 2-arrangements of $[n-1]$ and Dyck paths of semilength $n$. Such a Dyck path consists of upsteps $(1,1)$ and downsteps $(1,-1)$, starting from $(0,0)$, ending at $(2 n, 0)$ and never going below the $x$-axis. A Dyck path can be decomposed into shorter such paths, according to where it touches the $x$-axis. The part strictly between two consecutive such steps, the first an upstep from the axis, the latter a downstep to the axis, can be any Dyck path of the appropriate length, lifted one unit.

In a 312-avoiding permutation form of a 2-arrangement the positive letters to the right of any -1 must each be larger than all the positive letters preceding that -1 . The -1 s thus partition the positive letters into blocks (possibly empty), each block consisting of letters determined by its size and the number of positive letters to its right, and each block can contain an arbitrary 312-avoiding permutation of those letters. The 312-avoiding permutations of $[m]$ are well known to be in bijection with Dyck paths of semilength $m$ and so this structural decomposition is identical to that of Dyck paths described above. This correspondence pairs Dyck paths of semilength $n$ with arrangements of $[n-1]$ because we must prepend a -1 to ensure that the first block of positive letters is preceded by a -1 .

It follows that 2-arrangements of $[n-1]$ whose permutation form avoids 312 are in bijection with 312 -avoiding permutations of $[n]$. It was shown in [SW06, Thm. 3] that the number of permutations of a given multiset that avoid a pattern of length 3 is independent of the pattern. As we showed in Proposition 3, the set of $k$-arrangements for any $n$ and $k$ is a union of rearrangement classes, and the permutations of a multiset are precisely the rearrangement class of any word consisting of the letters of that multiset.

Finally, since the $-1 \mathrm{~s}$ in a 312-avoiding 2-arrangement of $[n-1]$ correspond bijectively to all but the last return to the $x$-axis of the corresponding Dyck path, the distribution of the number of $-1 \mathrm{~s}$ claimed follows from a well known fact about Dyck paths.

Conjecture 2. The number of 3-arrangements of $[n]$ whose permutation form avoids any single pattern of length 3 is $C(n+2)-2^{n}$.

Conjecture 3. The distribution of the number of descents on 2-arrangements of $[n-1]$ whose permutation form avoids any single one of the patterns 132, 213, 231 or 312, is given by the triangle sequence A108838 in [OEI], which counts, among other things, rooted ordered trees with $n$ non-root nodes and $k$ leaves, and has formula $\frac{2}{(n+1)}\left(\begin{array}{c}n+1 \\ k+2\end{array}\right)\left(\begin{array}{c}n-2 \\ k\end{array}\right)$.

Conjecture 4. The distribution of the number of ascents on 2-arrangements of $[n-1]$ whose permutation form avoids the pattern 123, is given by triangle sequence A236406 in [OEI], which counts 123-avoiding permutations of $[n]$ with $k$ peaks.

Given a $k$-arrangement $\mathfrak{a}$ as a permutation $\pi$ and a map $\phi: \operatorname{Fix}(\pi) \rightarrow[k]$, define the color-encoding of $\mathfrak{a}$ to be the $k$-colored permutation $(\pi, c)$ for which $c_{i}=\phi(i)$ if $i \in \operatorname{Fix}(\pi)$ and $c_{i}=0$ otherwise. We can then apply the statistics in Definition 5 to the color-encoding of a $k$-arrangement.

Conjecture 5. The statistics inv and maj in Definition 5 are equidistributed on $\mathrm{A}_{\mathrm{n}}^{\mathrm{k}}$ in the color encoding for any $n$ and $k$. The statistic des in that definition has the same distribution on $A_{n}^{k}$ as des on the permutation or derangement form for any $n$ and $k$.

The following also seems worthy of investigation.

Problem 1. Proposition 1 shows how the continued fraction $\mathcal{C}_{\mathbf{u}}$ and its ordinary generating function capture our fundamental statistics for permutations together with the coloring of fixed points in $k$-arrangements. Is there a specialization of $\mathcal{C}_{\mathbf{u}}$, or a similar continued fraction, that captures the distribution of the statistics on colored permutations in Definition 5 applied to $k$-arrangements? What about the statistics defined for the derangement and permutation forms in Definition 8 ? 


\section{The Central Bijection}

In this section we describe the bijective map $\eta: \mathcal{S}_{n} \rightarrow \mathcal{M}_{n}$, whose existence was asserted in Theorem 2. The map $\eta$ takes permutations, refined in terms of fourteen combinatorial statistics introduced in Definition 2, to Motzkin paths with labels as prescribed in Definition 3.

\subsection{Preliminaries}

We start by observing that when the parameters $a$ through $w$ are set to 1, each Motzkin path labeled as in Definition 3 carries unit weight. Since the same parameter setting in (3) gives $\alpha_{n}=2 n+1$ and $\beta_{n}=n^{2}$, it then follows easily (see e.g. [Fla80, Theorem 3B]) that $\mathcal{C}$ becomes the continued fraction expansion of the ordinary generating function for the sequence $(n !)$. In other words:

Lemma 2. The number of Motzkin paths $\mathcal{M}_{n}$ labeled as in Definition 3 is $n$ !.

To define the map $\eta$, it is convenient to introduce 'vectorized' versions of the permutation statistics of Definition 2.

Definition 10. Given $\sigma \in \mathcal{S}_{n}$ and $i \in[n]$, let

- inve $_{i}(\sigma)=\#\{x \in[n] \mid x<i<\sigma(i)<\sigma(x)\}$.

- $\operatorname{ninve}_{i}(\sigma)=\#\{x \in[n] \mid x<i<\sigma(x)<\sigma(i)\}$.

- $\operatorname{inva}_{i}(\sigma):=\#\{x \in[n] \mid x>i>\sigma(i)>\sigma(x)\}$.

- $\operatorname{ninva}_{i}(\sigma):=\#\{x \in[n] \mid x>i>\sigma(x)>\sigma(i)\}$.

- $\operatorname{iefp}_{i}(\sigma):=\#\{x \in[n] \mid x<i<\sigma(x)\}$.

Here the conditions on $x, i, \sigma(x), \sigma(i)$ in inve $e_{i}$ are the same as on $i, j, \sigma(i), \sigma(j)$ (in that order) in both ie and ile in Definition 2. (Recall that ile counts inversions among excedances where the rightmost of the two excedances is linked, so that (ie - ile) counts such inversions where the rightmost excedance is not linked.) Analogous considerations apply to inva ${ }_{i}$ and ninva ${ }_{i}$, whereas $\operatorname{iefp}_{i}$ will be applied only to fixed points $i$.

The structure of the Motzkin path $\eta(\sigma)$ closely depends on the type (linked vs. non-linked) of the excedances and anti-excedances in $\sigma$, as well as on the number of chains formed by these. Namely:

Definition 11. A sequence $i_{1}, i_{2}, \ldots, i_{p}$ of excedances forms a chain of excedances if

$$
i_{1}<\sigma\left(i_{1}\right)=i_{2}<\sigma\left(i_{2}\right)=i_{3}<\cdots<\sigma\left(i_{p-1}\right)=i_{p}<\sigma\left(i_{p}\right) .
$$

If $i_{1}, i_{2}, \ldots, i_{p}$ is a maximal chain of excedances (w.r.t. containment), then its starter is the excedance $i_{1} \mapsto \sigma\left(i_{1}\right)$. A chain of excedances $i_{1}, i_{2}, \ldots, i_{p}$ is said to span $z \in[n]$ if $i_{1}<z<\sigma\left(i_{p}\right)$.

A sequence $i_{1}, i_{2}, \ldots, i_{p}$ of anti-excedances forms a chain of anti-excedances if

$$
i_{1}>\sigma\left(i_{1}\right)=i_{2}>\sigma\left(i_{2}\right)=i_{3}>\cdots>\sigma\left(i_{p-1}\right)=i_{p}>\sigma\left(i_{p}\right) .
$$

The starter of a maximal such chain is the anti-excedance $i_{1} \mapsto \sigma\left(i_{1}\right)$. A chain of anti-excedances $i_{1}, i_{2}, \ldots, i_{p}$ is said to span $z \in[n]$ if $i_{1}>z>\sigma\left(i_{p}\right)$. 
Remark 4. Note that in a maximal chain of excedances all excedances are linked except for the starter, and likewise for a maximal chain of anti-excedances. Also, $x \in[n]$ is spanned by a chain of excedances or anti-excedances if the chain 'begins and 'ends' on different sides of $x$.

Lemma 3. For every $\sigma \in \mathcal{S}_{n}$ and $m \in[n]$ the number of non-linked excedances in $[m]$ is at least as large as the number of non-linked anti-excedances in $[\mathrm{m}]$. Moreover, the total number of non-linked excedances in $\sigma$ is equal to the total number of non-linked anti-excedances.

Proof. Let $i$ be any non-linked anti-excedance in $\sigma$, so $\sigma(i)<i$. This implies that $j=\sigma^{-1}(i)$ is an excedance, so $j<i$, since otherwise $i$ would be a linked anti-excedance. Now, $j=\sigma^{-1}(i)$ is an excedance whose maximal chain of excedances starts with a non-linked excedance $k \leq j$. We associate the non-linked anti-excedance $i$ to this non-linked excedance $k$.

Two different maximal chains of excedances cannot intersect in any $i \in[n]$, since that would imply that $\sigma(i)$ had two different values for some $i$. Thus, each non-linked anti-excedance is associated to a unique non-linked excedance preceding it, which shows that reading from left to right we can never have more non-linked anti-excedances than non-linked excedances.

To show that a permutation $\sigma$ has equally many non-linked excedances and non-linked antiexcedances in total, apply the above argument to the reverse complement of $\sigma$, whose two-line diagram is obtained by reflecting the diagram for $\sigma$ in its vertical bisector (and reversing the numbers to have them increase from left to right). That transformation clearly interchanges non-linked excedances and non-linked anti-excedances.

\subsection{Constructing $\eta$}

To define the map $\eta: \mathcal{S}_{n} \rightarrow \mathcal{M}_{n}$ of Theorem 2, we begin by associating to each permutation $\sigma \in \mathcal{S}_{n}$ a path in $\mathbb{R}^{2}$ starting at $(0,0)$ and composed of level steps, NE upsteps, and SE downsteps as follows. For $i=1, \ldots, n$, the $i$ th step in the path is an upstep if $i \mapsto \sigma(i)$ is a non-linked excedance, a downstep if $i \mapsto \sigma(i)$ is a non-linked anti-excedance, and a level step otherwise. By Lemma 3, the path will terminate on the abscissa without ever falling below it. The paths obtained are therefore Motzkin paths.

Fix $\sigma \in \mathcal{S}_{n}$. If an excedance $i \mapsto \sigma(i)$ is linked, the $i$-th step is a level step labeled

$$
a^{\text {inve }_{i}(\sigma)} b^{\text {ninve }_{i}(\sigma)} s
$$

and, otherwise, if the excedance is not linked, the $i$-th step is an upstep labeled

$$
c^{\text {inve }_{i}(\sigma)} d^{\text {ninve }_{i}(\sigma)} p .
$$

Similarly, if an anti-excedance $i \mapsto \sigma(i)$ is linked, the $i$-th step is a level step labeled

$$
f^{\operatorname{inva}_{i}(\sigma)} g^{\text {ninva }_{i}(\sigma)} t
$$

and, otherwise, if the anti-excedance is not linked, the $i$-th step is a downstep labeled

$$
h^{\operatorname{inva}_{i}(\sigma)} \ell^{\text {ninva }_{i}(\sigma)} r .
$$

Finally, if $i$ is a fixed point in $\sigma$ then the $i$-th step in the path is a level step labeled

$$
u \cdot w^{\operatorname{iefp}_{i}(\sigma)} .
$$


The following identities are elementary:

$$
\prod_{\substack{i \in[n] \\ \sigma^{-1}(i)<i<\sigma(i)}} a^{\text {inve }_{i}(\sigma)} b^{\text {ninve }_{i}(\sigma)}=a^{\text {ile }(\sigma)} b^{\text {nile }(\sigma)}
$$

and

$$
\prod_{\substack{i \in[n] \\ i<\min \left(\sigma(i), \sigma^{-1}(i)\right)}} c^{\text {inve }_{i}(\sigma)} d^{\text {ninve }_{i}(\sigma)}=c^{\text {ie }(\sigma)-\text { ile }(\sigma)} d^{\text {nie }(\sigma)-\text { nile }(\sigma)} .
$$

Analogous statements hold for the anti-excedances, and for the fixed points (with iefp), showing that the weight of a labeled path $\eta(\sigma)$ is as stated in the following theorem.

Theorem 3. The map $\eta: \mathcal{S}_{n} \rightarrow \mathcal{M}_{n}$ is a bijection with the property that, for $M=\eta(\sigma)$,

$$
\begin{aligned}
\operatorname{wt}(M)= & a^{\text {ile }(\sigma)} b^{\text {nile }(\sigma)} c^{\text {ie }(\sigma)-\text { ile }(\sigma)} d^{\text {nie }(\sigma)-\operatorname{nile}(\sigma)} f^{\text {ilae }(\sigma)} g^{\text {nilae }(\sigma)} h^{\text {iae }(\sigma)-\text { ilae }(\sigma)} \ell^{\text {niae }(\sigma)-\text { nilae }(\sigma)} \\
& \times p^{\operatorname{exc}(\sigma)-\operatorname{le}(\sigma)} r^{\operatorname{aexc}(\sigma)-\operatorname{lae}(\sigma)} s^{\operatorname{le}(\sigma)} t^{\operatorname{lae}(\sigma)} u^{\mathrm{fp}(\sigma)} w^{\operatorname{iefp}(\sigma)}
\end{aligned}
$$

The proof of Theorem 3 hinges on the following lemma.

Lemma 4. Suppose $A$ and $B$ are finite subsets of $\mathbb{N}$ with $\# A=\# B$ and let $\phi, \psi: A \rightarrow B$ be bijections such that, for every $i \in A$, we have $i<\phi(i), i<\psi(i)$, and

$$
\#\{x \in A \mid x<i<\phi(i)<\phi(x)\}=\#\{x \in A \mid x<i<\psi(i)<\psi(x)\}
$$

Then $\phi=\psi$.

Proof. We proceed by induction on $\kappa:=\# A=\# B$. Let $A=\left\{i_{1}, \ldots, i_{\kappa}\right\}$ and $B=\left\{j_{1}, \ldots, j_{\kappa}\right\}$, with $i_{1}<\cdots<i_{\kappa}$ and $j_{1}<\cdots<j_{\kappa}$. Then it is easy to verify that $(25)$ implies $\phi\left(i_{\kappa}\right)=\psi\left(i_{\kappa}\right)=j_{\lambda}$ where

$$
\begin{aligned}
\lambda & =\kappa-\#\left\{x \in A \mid x<i_{\kappa}<\phi\left(i_{\kappa}\right)<\phi(x)\right\} \\
& =\kappa-\#\left\{x \in A \mid x<i_{\kappa}<\psi\left(i_{\kappa}\right)<\psi(x)\right\}
\end{aligned}
$$

Now removing $i_{\kappa}$ from $A$ and $j_{\lambda}$ from $B$, and restricting $\phi$ and $\psi$ accordingly, the equality in (25) still holds for the modified sets and bijections. Thus, by induction, $\phi=\psi$.

Proof of Theorem 3. Since $\left|\mathcal{M}_{n}\right|=n$ ! by Lemma 2, it suffices to show that $\eta$ is injective. To this end, let $\sigma, \sigma^{\prime} \in \mathcal{S}_{n}$ be permutations mapped to the same labeled Motzkin path $M=\eta(\sigma)=\eta\left(\sigma^{\prime}\right)$, and we will show that this forces $\sigma=\sigma^{\prime}$.

Let $\mathcal{F}$ be the set of all positions of the level steps in $M$ whose label is of the form $u w^{i}$. Let $\mathcal{E}_{0}$ be the set of all positions of the upsteps in $M, \mathcal{E}_{1}$ the set of all positions of the level steps in $M$ whose label is of the form $a^{i} b^{j} s$ and $\mathcal{E}=\mathcal{E}_{0} \cup \mathcal{E}_{1}$. Similarly, let $\mathcal{A}_{0}$ be the set of all positions of the downsteps in $M, \mathcal{A}_{1}$ the set of all positions of the level steps in $M$ whose label is of the form $f^{i} g^{j} t$ and $\mathcal{A}=\mathcal{A}_{0} \cup \mathcal{A}_{1}$. Notice that the sets $\mathcal{F}, \mathcal{E}_{0}, \mathcal{E}_{1}, \mathcal{A}_{0}, \mathcal{A}_{1}$ are disjoint and their union is $[n]$. Also, $\mathcal{E}_{0}$ equals the set of non-linked excedances in $\sigma$ and $\sigma^{\prime}, \mathcal{E}_{1}$ equals the set of linked excedances in $\sigma$ and $\sigma^{\prime}$, and similarly for $\mathcal{A}_{0}, A_{1}$ and anti-excedances, whereas $\mathcal{F}$ is the set of fixed points in $\sigma$ and $\sigma^{\prime}$.

Recalling that under the map $\eta$ fixed points in the permutation map to $u w^{i}$-labeled level steps, we have that $\sigma(i)=\sigma^{\prime}(i)=i$ for all $i \in \mathcal{F}$. Next we show that $\sigma(\mathcal{E})=\sigma^{\prime}(\mathcal{E})$. Note first that 
every linked excedance is the image, under $\sigma$, of an excedance, so $\mathcal{E}_{1}$ is contained in $\sigma(\mathcal{E})$. Also, a non-linked excedance cannot belong to $\sigma(\mathcal{E})$, so the remainder of $\sigma(\mathcal{E})$ consists of anti-excedances. These are precisely all the non-linked anti-excedances, because they are all images of excedances, by definition, and the linked anti-excedances are not. The same holds for $\sigma^{\prime}$ and so we have that

$$
\sigma(\mathcal{E})=\sigma^{\prime}(\mathcal{E})=\mathcal{E}_{1} \cup \mathcal{A}_{0} .
$$

Let $\mathcal{E}=\left\{i_{1}, \ldots, i_{\kappa}\right\}$ with $i_{1}<\cdots<i_{\kappa}$ and $\sigma(\mathcal{E})=\left\{j_{1}, \ldots, j_{\kappa}\right\}$ with $j_{1}<\cdots<j_{\kappa}$. Now, since $\sigma$ and $\sigma^{\prime}$ map to the same labeled Motzkin path we must have inve $i(\sigma)=$ inve $_{i}\left(\sigma^{\prime}\right)$ for all $i$. This implies that $\sigma$ and $\sigma^{\prime}$ satisfy the conditions on $\phi$ and $\psi$ in Equation (25), with $A$ in Lemma 4 being $\mathcal{E}$. Thus, Lemma 4 implies that $\sigma(\mathcal{E})=\sigma^{\prime}(\mathcal{E})$. (Note that ninve is the number of $x \in[n]$ such that $x<i_{\kappa}<\sigma(x)<\sigma\left(i_{\kappa}\right)$ and that the assignment $\sigma\left(i_{\kappa}\right)=\sigma^{\prime}\left(i_{\kappa}\right)=j_{\kappa-\text { inve }}$ implied by Lemma 4 is necessarily consistent with this.)

Finally, taking the reverse complements of $\sigma$ and $\sigma^{\prime}$ or, equivalently, using the analogue of Lemma 4 in which all the inequalities are reversed, similarly shows that $\sigma$ and $\sigma^{\prime}$ also agree on the set $\mathcal{A}$. Hence, $\sigma=\sigma^{\prime}$, so $\eta$ is injective and thus bijective.

Remark 5. Note that in the proof of Theorem 3, no mention is made of the values of $\operatorname{iefp}_{i}(\sigma)$. The reason is that a permutation is determined uniquely by a much smaller set of statistics than those present in the labels of the corresponding Motzkin path. It is easy to see that knowing the excedance bottoms and tops $(i$ and $\sigma(i)$ for all excedances $i)$, together with the set of fixed points and the vector statistics inve ${ }_{i}$ and inva $a_{i}$ for all $i$, is enough to determine a permutation.

\subsection{Some properties of $\eta$}

It follows from the definition of the map $\eta$ that the height of the left end of the $i$ th step in the path $\eta(\sigma)$ equals the number of non-linked excedances preceding the $i$ th place in $\sigma$ minus the number of preceding non-linked anti-excedances, since only these incur a height difference. Below we give a different description of the height of the $i$ th step, in terms of statistics derived from those in Definition 10.

Definition 12. Given $\sigma \in \mathcal{S}_{n}$ let

- $\operatorname{prex}_{i}(\sigma)=\#\{x \in[n] \mid x<i<\sigma(x)\}$,

- fola $_{i}(\sigma)=\#\{x \in[n] \mid \sigma(x)<i<x\}$.

Note that if $i$ is an excedance then $\operatorname{prex}_{i}(\sigma)=\operatorname{inve}_{i}(\sigma)+\operatorname{ninve}_{i}(\sigma)$ and if $i$ is an anti-excedance then fola $_{i}(\sigma)=\operatorname{inva}_{i}(\sigma)+\operatorname{ninva}_{i}(\sigma)$. If $i$ is a fixed point then $\operatorname{prex}_{i}(\sigma)$ counts the same indices $x$ as it does for an excedance. Note also that $\operatorname{prex}_{i}(\sigma)$ is the number of excedances $x$ preceding $i$ such that $\sigma(x)>i$ and that fola $i(\sigma)$ is the number of anti-excedances $x$ following $i$ such that $\sigma(x)<i$.

Proposition 7. Given $\sigma \in \mathcal{S}_{n}$ let $M$ be the labeled Motzkin path $M=\eta(\sigma)$. Then, for $i \in[n]$,

(i) if $i$ is a linked excedance then the ith step in $M$ is a level step at height $\operatorname{prex}_{i}(\sigma)+1$.

(ii) if $i$ is a non-linked excedance then the ith step in $M$ is an upstep starting at height $\operatorname{prex}_{i}(\sigma)$.

(iii) if $i$ is a linked anti-excedance then the ith step in $M$ is a level step at height fola ${ }_{i}(\sigma)+1$.

(iv) if $i$ is a non-linked anti-excedance then the ith step in $M$ is a downstep starting at height fola $_{i}(\sigma)$. 
(v) If $i$ is a fixed point then the ith step in $M$ is a level step whose height is $\operatorname{prex}_{i}(\sigma)$.

Proof. Recall that the starter of each maximal chain of excedances is a non-linked excedance, corresponding to an upstep, while any remaining excedances in the chain are necessarily linked, corresponding to level steps. Furthermore, each maximal chain of excedances terminates in a nonlinked anti-excedance $j$, corresponding to a downstep, namely, where $\sigma^{-1}(j)$ is the last excedance in the chain. Conversely a non-linked anti-excedance always terminates some chain of excedances.

Thus, the number of non-linked excedances (strictly) preceding an excedance $i$ equals the number of maximal chains of excedances starting before $i$, and the number of non-linked anti-excedances preceding $i$ equals the number of maximal chains of excedances that end before $i$. The difference between these two numbers is the number of maximal chains of excedances that span $i$, but this difference is also the height of the $i$ th step of $M$, as pointed out just before Definition 12. Each such chain spanning $i$ has a rightmost excedance $x$ strictly preceding $i$ if $i$ is a non-linked excedance, which thus satisfies $x<i<\sigma(x)$, and those inequalities are satisfied by precisely one excedance $x$

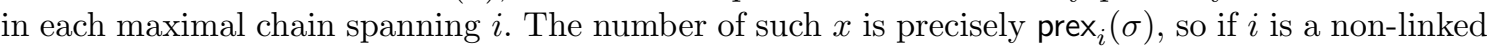
excedance the $i$ th step starts at height $\operatorname{prex}_{i}(\sigma)$. If $i$ is a linked excedance then the maximal chain that $i$ belongs to also spans $i$, and so the $i$ th step (which is level) is at height $\operatorname{prex}_{i}(\sigma)+1$.

The argument in the case of anti-excedances is analogous, since reversing a path $\eta(\sigma)$, which corresponds to taking the reverse complement of $\sigma$ (see proof of Lemma 3), turns excedances into anti-excedances and vice versa, and preserves linking.

Finally, if $i$ is a fixed point then the number of chains spanning $i$ equals the number of elements $x \in[n]$ satisfying $x<i<\sigma(x)$, so the height of the corresponding level step is $\operatorname{prex}_{i}(\sigma)$, the argument being identical to that for non-linked excedances.

\subsection{Ties to previous bijections}

As mentioned before, several authors have defined statistics on permutations that have then been used to construct bijections to Motzkin paths, labeled to reflect those statistics [FV79, FZ90, Bia93, SS96, CSZ97, Ran98]. These statistics essentially come in two flavors, being based either on excedances, as in the present paper and [FZ90, Bia93, Ran98] or descents as in [FV79, SS96], a bijection in [CSZ97] translating between these. In each of these two groups, each permutation is mapped to the same Motzkin path, but labels differ. We give here, without proofs, some indication of the similarities and differences between these labelings, showing in the process that the bijection introduced in this paper cannot be recovered from previous published work.

First, it is helpful to consider a minor modification of the setup in [SS96], where Simion and Stanton partition a permutation into ascent blocks (which they call runs), maximal contiguous increasing segments in a a permutation. To facilitate the discussion here, we will follow [CSZ97] and partition into descent blocks, which are decreasing rather than increasing. A bijection translating the ascent-based framework in [SS96] to the descent-based one in [CSZ97] is taking reverse of each permutation, that is, sending a permutation $a_{1} a_{2} \ldots a_{n}$ to $a_{n} \ldots a_{2} a_{1}$. For example, the reverse of the permutation 36-4-125 is 521-4-63, where we separate the ascent/descent blocks by dashes. We then say that the first letter in a non-singleton block is an opener and its last letter a closer. Other letters in non-singleton blocks are insiders (continuators in [SS96]). In 521-4-63 the openers are 5,6 , the closers are 1,3 , whereas 2 is an insider and 4 a singleton. Clearly, reversing a permutation interchanges openers with closers and preserves insiders and singletons, and the vector statistics $\operatorname{lsg}_{i}$ and $\mathrm{rsg}_{i}$ in[SS96, Def. 2.1] are also interchanged. What we say about the statistics of Simion and Stanton below thus applies to the reversed version, with descent blocks instead of ascent blocks. 
In [CSZ97, Section 3] a bijection $\Phi$ on permutations is presented that translates the above 'descent based' classes of letters into 'excedance based' classes. Namely, in a permutation $\pi=a_{1} a_{2} \ldots a_{n}$, written in one line notation, if $a_{i}>i$ then $a_{i}$ is an 'excedance top' and $i$ and 'excedance bottom', whereas if $a_{i} \leq i$ then $a_{i}$ is a 'non-excedance top' and $i$ a 'non-excedance bottom'. When $\Phi$ is applied to a permutation, such as 521-3-6-74, which $\Phi$ maps to 2715364 , we get the following correspondences between these four classes, where the last column gives the classes used in the present paper (the singletons 3 and 6 mapping to a linked anti-excedance and a fixed point, respectively):

\begin{tabular}{ccc} 
in $\sigma[\mathrm{SS} 96]$ & in $\Phi(\sigma)[\mathrm{CSZ97}]$ & in $\Phi(\sigma)$ (present) \\
\hline opener & excedance top \& non-excedance bottom & non-linked anti-excedance \\
\hline closer & non-excedance top \& excedance bottom & non-linked excedance \\
\hline insider & excedance top \& excedance bottom & linked excedance \\
\hline singleton & non-excedance top \& non-excedance bottom & linked anti-excedance/fixpt \\
\hline
\end{tabular}

It turns out that all the bijections in the papers mentioned above translate a permutation to the same unlabeled Motzkin path, modulo the bijection $\Phi$ and/or some trivial bijections such as reversing a permutation. The bijection $\Phi$ also translates between the various statistics studied in [SS96] and [CSZ97], respectively, where the inversion statistics in the latter correspond to the statistic rsg in the former, which counts occurrences of the vincular pattern 2-31. These statistics determine the labeling of the steps in the Motzkin paths that respectively correspond to permutations in each case. In [SS96] Simion and Stanton also incorporate the 'mirror' statistic Isg, which counts occurrences of 31-2. This latter statistic can be incorporated in Theorem 10 in [CSZ97] (as was done in [CM02, Theorem 22]), by replacing the brackets $[\cdot]_{p}$ with $[\cdot]_{p, p^{\prime}}$, and including the corresponding mirror images of the inversion statistics in $f(x, p, q)$ in [CSZ97].

Since fixed points are treated as non-excedance tops and non-excedance bottoms in [CSZ97], and thus not distinguished from linked anti-excedances, both correspond to singletons in $\Phi^{-1}(\sigma)$. This setup thus differs from that of the present paper and in particular leads to the asymmetry in the continued fractions that 'mix' the brackets $[n]$ and $[n+1]$ in both [SS94] and [CSZ97]. As Simion and Stanton [SS94] base their statistics on the one-line notation of ascent blocks, and classify a letter of a permutation according to the relative sizes of its predecessor and follower, it is impossible to accommodate more than four distinct classes of letters.

There is nevertheless a way to characterize what happens to fixed points in a permutation $\sigma$ under $\Phi^{-1}$. Namely, a fixed point $i$ in $\sigma$ corresponds in $\Phi^{-1}(\sigma)$ to a $\operatorname{singleton} i$ with $\operatorname{lsg}(i)=0$, that is, a singleton that is not the 2 in any occurrence of 31-2. This corresponds to the fact that a fixed point in $\sigma$ can not form an inversion with a non-excedance preceding it.

In [Ran98], Randrianarivony presents a generating function that expands the one in [SS96] by adding five statistics: Orsg, which is evaluated on each of the four types of letters (openers, closers, etc.), and $D$. The statistic $D$ is a linear combination of other statistics in the generating function, and so does not add information. The Orsg statistics, on the other hand, are not vector statistics, since they count, for each type of letter, the number of such letters $i$ in $\sigma$ with $\operatorname{rsg}_{i}(\sigma)=0$. Because of our reversal here of the Simion-Stanton setup, our statistic $\operatorname{lsg}_{i}$ corresponds to rsg ${ }_{i}$ in the paper by Randrianarivony. Thus, his pointing out that Orsg applied to the singletons counts double descents $i$ with $\operatorname{rsg}(i)=0$, is equivalent to our claim about fixed points in the preceding paragraph, because a double descent in the ascent-based setup is a singleton, between two ascent blocks.

As for the bijections of Françon and Viennot [FV79] and Foata and Zeilberger [FZ90], it is explained in [CSZ97, Section 5] how they are equivalent, via the bijection $\Phi$. 
In short, separating fixed points as a class of their own — as we have done here - in defining the vector statistics at the heart of these different schemes, is not possible within the schemes of Simion-Stanton [SS96] and Randrianarivony [Ran98]. The same is true of the other schemes mentioned above, which all are equivalent to that of Simion and Stanton, as previously explained.

In the recent preprint [SZ] Sokal and Zeng consider both 'records' (left-to-right maxima) and 'antirecords' (right-to-left minima), and partition letters in a permutation into four classes depending on these attributes. They also partition letters into 'cycle peaks', 'cycle valleys', 'cycle double rises', 'cycle double falls' and fixed points, the double rises and double falls corresponding to our linked excedances and linked anti-excedances, respectively, the other two 'cycle' classes to our non-linked cases of these. By considering intersections of these two kinds of classes, they come up with a partitioning into ten distinct categories. In addition to this they incorporate nine statistics that are refinements of the crossings and nestings defined by Corteel [Cor07], combinations of which can be shown to equal our inversion statistics in items 6-14, Definition 2.

It is worth comparing our labels of steps in Motzkin paths, in (18)-(22) above, with the corresponding labels of the bijection of Foata and Zeilberger [FZ90], as described in [CSZ97, Section 5]. In [CSZ97] the label assigned to each excedance $i$ is the number of excedances $j$ preceding $i$ where $\sigma(j)>\sigma(i)$, that is, the label of an excedance is the 'inversion bottom number' of that excedance among all excedances. This agrees exactly with our label inve ${ }_{i}(\sigma)$, which is independent of whether the excedance $i$ is linked. In [CSZ97], the label assigned to each non-excedance (which includes fixed points), is the number of non-excedances following it and smaller than it, which agrees with our inva only in the case of anti-excedances.

While a fixed point is classified as a non-excedance in [CSZ97], and the label associated to it therefore depends on smaller non-excedances following it, here we label a fixed point $i$ by $u w^{\operatorname{iefp}_{i}(\sigma)}$, which thus depends on excedances preceding it. The labeling in [CSZ97] consequently differs from ours. Our labeling is also different from Biane's labeling in [Bia93], which in [CSZ97] is shown to be closely related to the labeling described in [CSZ97]. Finally, the labeling of Simion-Stanton in [SS94], after the translation applying $\Phi$ to the reverse of each permutation in their setting, is also different from ours, as is inevitable since they do not distinguish between what after that translation become linked anti-excedances and fixed points, respectively.

Crucially, our labeling of excedances and anti-excedances is independent of fixed points in a permutation, which is not the case for the labeling in [CSZ97] nor, by the equivalences we have described above, for the other bijections mentioned above (except for the recent preprint [SZ]). Specifically, underlying all these results is some scheme for partitioning letters that form a permutation, which is at the core of each construction. While the aforementioned papers (again excluding [SZ]) can only accommodate a partition into four classes, we have a separate, fifth class, containing the fixed points. This distinction allows for a greater variety of special cases, extensions to signed and colored permutations, as well as to a natural notion of $k$-arrangements.

\section{Acknowledgements}

We would like to thank Roman Stocker and Martin Ackermann for their hospitality at ETH Zürich where some of this work was done. This collaboration started through a LMS Celebrating New Appointments Grant and was further enabled by the Lancaster Mathematics \& Statistics Visitor Fund. Thanks also to Alan Sokal and Jiang Zeng, for pointing out the paper by Randrianarivony [Ran98], and for kindly sharing a draft of their manuscript [SZ] with us as ours was being finalized. Lastly, we are indebted to an anonymous referee whose careful reading and valuable suggestions improved this paper, especially with regard to the streamlining of the proof of Theorem 3 via Lemma 4. 


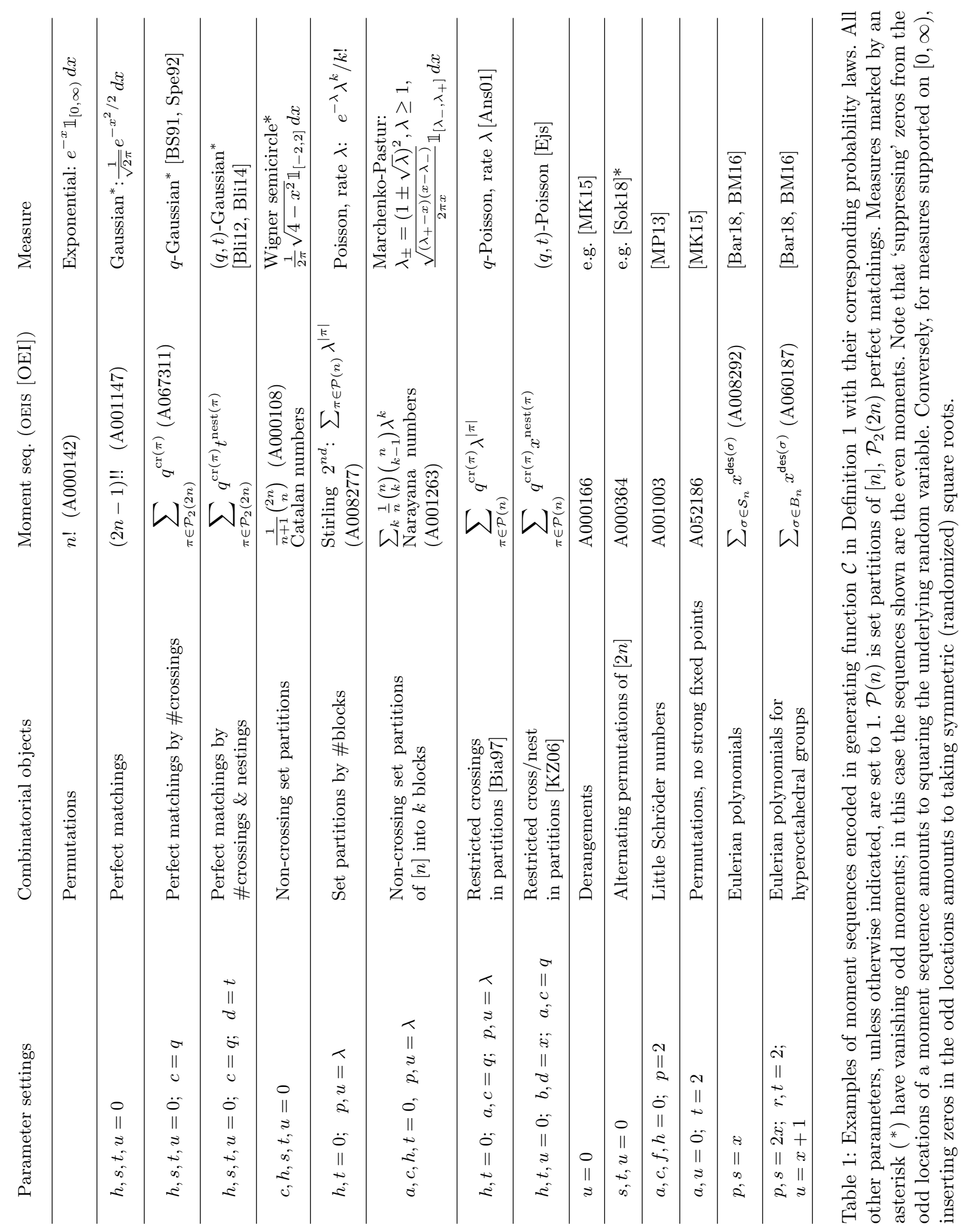


Orthogonal

polynomial sequence

Normaliz. Parameters $(a, b, c, d, f, g, h, \ell, p, r, s, t, u)$ in $\mathcal{C}$ with $w=0$ in all cases

\begin{tabular}{lll}
\hline Discrete $q$-Hermite II & $(14.29 .5)$ & $\left(0,0,0, q^{-2}, 0,0,1, q, q^{-1}, 1-q, 0,0,0\right)$ \\
Discrete $q$-Hermite I & $(14.28 .4)$ & $(0,0,0, q, 0,0,1, q, 1,1-q, 0,0,0)$ \\
Stieltjes-Wigert & $(14.27 .4)$ & $\left(0, q^{-2}, 0, q^{-4}, 0, q^{-1}, 1, q, q^{-3}, 1-q,(1+q) q^{-3},-q^{-1}, q^{-1}\right)$ \\
Continuous $q$-Hermite & $(14.26 .4)$ & $(0,0,0,1,0,0,1, q, 1 / 4,1-q, 0,0,0)$ \\
Al-Salam-Carlitz II & $(14.25 .4)$ & $\left(0, q^{-1}, 0, q^{-2}, 0,0,1, q, a q^{-1}, 1-q,(a+1) q^{-1}, 0, a+1\right)$ \\
Al-Salam-Carlitz I & $(14.24 .4)$ & $(0, q, 0, q, 0,0,0, q, a, 1-q,(a+1) q, 0, a+1)$ \\
$q$-Charlier $(a=1)$ & $(14.23 .4)$ & $\left(0, q^{-2}, 0, q^{-4}, 0, q^{-2}, 1, q^{2}, q^{-3}, 1-q^{2}, q^{-3}, q^{-2}, 1+q^{-1}\right)$ \\
$q$-Laguerre $(\alpha=0)$ & $(14.21 .6)$ & $\left(0, q^{-2}, q^{-4}, q^{-3}, 0, q^{-1}, 1, q, q^{-3}-q^{-2}, 1-q,(1+q) q^{-3},-2 q^{-1},(1-q) q^{-1}\right)$ \\
Little $q$-Lag./Wall $(a=1)$ & $(14.20 .4)$ & $\left(0, q, q, q^{2}, 0, q^{2}, q, q^{2}, 1-q, q(1-q), 2 q,-(1+q) q^{2}, 1-q\right)$ \\
Cont. $q$-Laguerre $(\alpha=0)$ & $(14.19 .4)$ & $\left(0, q, 1, q, 0,0,1, q,(1-q) / 2,(1-q) / 2,(1+\sqrt{q}) q^{5 / 4} / 2,0, q^{1 / 4}\left(1+q^{1 / 2}\right) / 2\right)$ \\
Cont. big $q$-Hermite & $(14.18 .5)$ & $(0, q, 1, q, 0,0,1,0,(1-q) / 4,1, a q / 2,0, a / 2)$ \\
$q$-Meixner $(b=c=1)$ & $(14.13 .4)$ & $\left(0, q^{-2}, q^{-4}, q^{-3}, q^{-2}, q^{-1}, 1, q^{2}, q^{-3}-q^{-2}, 1-q^{2}, q^{-3}, q^{-2}-q^{-1}, q^{-1}\right)$ \\
Big $q$-Laguerre $(a=-b=1)$ & $(14.11 .4)$ & $\left(0, q^{2}, q, q^{2}, 0, q, 1, q^{2}, q^{2}(1-q), 1-q^{2}, q^{3}(1+q),-q^{2}, q^{2}\right)$ \\
$q$-Meixner-Pollaczek $(a=\sqrt{q})$ & $(14.9 .4)$ & $\left(0, q, 1, q, 0,0,1, q,(1-q) / 2,(1-q) / 2, q^{3 / 2} \cos (\phi), 0, \sqrt{q} \cos (\phi)\right)$ \\
Al-Salam-Chihara $(a b=q)$ & $(14.8 .5)$ & $(0, q, 1, q, 0,0,1, q,(1-q) / 2,(1-q) / 2,(a+b) q / 2,0,(a+b) / 2)$ \\
Continuous dual $q$-Hahn & $(14.3 .5)$ & $\left(0, q^{2}, 1, q^{2}, 0, q^{2}, 1, q^{2},\left(1-q^{2}\right) / 2,\left(1-q^{2}\right) / 2, a^{-1} q^{4} / 2, a q^{2} / 2,\left(a^{2}+q^{2}\right) / 2 a\right)$
\end{tabular}

$(a b=-q, a c=q, b c=-q)$

Table 2: Examples of orthogonal polynomial families in $q$-Askey scheme encompassed by $\mathcal{C}$ in Definition 1. References for normalized recurrences are to equations in [KLS10]. Choice of parameters in $\mathcal{C}$ is generally non-unique.

\section{References}

[Ans01] Michael Anshelevich. Partition-dependent stochastic measures and q-deformed cumulants. Doc. Math., 6:343-384 (electronic), 2001.

[Bar18] Paul Barry. On a transformation of Riordan moment sequences. J. Integer Seq., 21(7):Art. 18.7.1, 19, 2018.

[BBEP17] David Bevan, Robert Brignall, Andrew Elvey Price, and Jay Pantone. Staircases, dominoes, and the growth rate of 1324-avoiders. Electron. Notes Disc. Math., (61):123-129, 2017.

[BEH15] Marek Bożejko, Wiktor Ejsmont, and Takahiro Hasebe. Fock space associated to Coxeter groups of type B. J. Funct. Anal., 269(6):1769-1795, 2015.

[BEH17] Marek Bożejko, Wiktor Ejsmont, and Takahiro Hasebe. Noncommutative probability of type D. Internat. J. Math., 28(2):1750010, 30, 2017.

[BGM14] Eli Bagno, David Garber, and Toufik Mansour. On the group of alternating colored permutations. Electron. J. Combin., 21(2):Paper 2.29, 28, 2014.

[Bia93] Philippe Biane. Permutations suivant le type d'excédance et le nombre d'inversions et interprétation combinatoire d'une fraction continue de Heine. European J. Combin., 14(4):277-284, 1993.

[Bia97] Philippe Biane. Some properties of crossings and partitions. Discrete Math., 175(13):41-53, 1997. 
[Bli12] Natasha Blitvić. The (q,t)-Gaussian process. J. Funct. Anal., 263(10):3270-3305, 2012.

[Bli14] Natasha Blitvić. Two-parameter non-commutative central limit theorem. Ann. Inst. Henri Poincaré Probab. Stat., 50(4):1456-1473, 2014.

[BM16] Anna Borowiec and Wojciech Młotkowski. New Eulerian numbers of type D. Electron. J. Combin., 23(1):Paper 1.38, 13, 2016.

[BS91] Marek Bożejko and Roland Speicher. An example of a generalized Brownian motion. Comm. Math. Phys., 137(3):519-531, 1991.

[BS94] Marek Bożejko and Roland Speicher. Completely positive maps on Coxeter groups, deformed commutation relations, and operator spaces. Math. Ann., 300(1):97-120, 1994.

[CGZJ18] Andrew R. Conway, Anthony J. Guttmann, and Paul Zinn-Justin. 1324-avoiding permutations revisited. Adv. in Appl. Math., 96:312-333, 2018.

[Cla01] Anders Claesson. Generalized pattern avoidance. European J. Combin., 22(7):961-971, 2001.

[CM02] Anders Claesson and Toufik Mansour. Counting occurrences of a pattern of type $(1,2)$ or $(2,1)$ in permutations. Adv. in Appl. Math., 29(2):293-310, 2002.

[Com74] Louis Comtet. Advanced combinatorics. D. Reidel Publishing Co., Dordrecht, enlarged edition, 1974. The art of finite and infinite expansions.

[Cor07] Sylvie Corteel. Crossings and alignments of permutations. Adv. in Appl. Math., 38(2):149-163, 2007.

[CSSW12] S. Corteel, R. Stanley, D. Stanton, and L. Williams. Formulae for Askey-Wilson moments and enumeration of staircase tableaux. Trans. Amer. Math. Soc., 364(11):60096037, 2012.

[CSZ97] Robert J. Clarke, Einar Steingrímsson, and Jiang Zeng. New Euler-Mahonian statistics on permutations and words. Adv. in Appl. Math., 18(3):237-270, 1997.

[CW11] Sylvie Corteel and Lauren K. Williams. Tableaux combinatorics for the asymmetric exclusion process and Askey-Wilson polynomials. Duke Math. J., 159(3):385-415, 2011.

[dMSW95] Anne de Médicis, Dennis Stanton, and Dennis White. The combinatorics of $q$-Charlier polynomials. J. Combin. Theory Ser. A, 69(1):87-114, 1995.

[dMV94] Anne de Médicis and Xavier G. Viennot. Moments des $q$-polynômes de Laguerre et la bijection de Foata-Zeilberger. Adv. in Appl. Math., 15(3):262-304, 1994.

[Egg15] Eric S. Egge. Defying God: the Stanley-Wilf conjecture, Stanley-Wilf limits, and a twogeneration explosion of combinatorics. In A century of advancing mathematics, pages 65-82. Math. Assoc. America, Washington, DC, 2015.

[Ehr00] Richard Ehrenborg. The Hankel determinant of exponential polynomials. Amer. Math. Monthly, 107(6):557-560, 2000.

[Ejs] Wiktor Ejsmont. Poisson type operators on the Fock space of type B and in the Blitvic model. J. Operator Theory, in press, arXiv:1811.02675. 
[Eli17] Sergi Elizalde. Continued fractions for permutation statistics. Discrete Math. Theor. Comput. Sci., 19(2):Paper No. 11, 24, 2017.

[EN03] Sergi Elizalde and Marc Noy. Consecutive patterns in permutations. Adv. in Appl. Math., 30(1-2):110-125, 2003. Formal power series and algebraic combinatorics (Scottsdale, AZ, 2001).

[EP18] Andrew Elvey-Price. Selected problems in enumerative combinatorics: permutation classes, random walks and planar maps. Thesis (Ph.D.)-University of Melbourne, 2018.

[FHL20] Shishuo Fu, Guo-Niu Han, and Zhicong Lin. $k$-Arrangements, Statistics, and Patterns. SIAM J. Discrete Math., 34(3):1830-1853, 2020.

[Fla80] P. Flajolet. Combinatorial aspects of continued fractions. Discrete Math., 32(2):125$161,1980$.

[FS70] Dominique Foata and Marcel-P. Schützenberger. Théorie géométrique des polynômes eulériens. Lecture Notes in Mathematics, Vol. 138. Springer-Verlag, Berlin-New York, 1970.

[FV79] Jean Françon and Gérard Viennot. Permutations selon leurs pics, creux, doubles montées et double descentes, nombres d'Euler et nombres de Genocchi. Discrete Math., 28(1):21-35, 1979.

[FZ90] Dominique Foata and Doron Zeilberger. Denert's permutation statistic is indeed EulerMahonian. Stud. Appl. Math., 83(1):31-59, 1990.

[Ham20] Hans Hamburger. Über eine Erweiterung des Stieltjesschen Momentenproblems. Math. Ann., (I) 81: 235-319, 1920; (II) 82: 120-164, 1920; (III) 82: 168-187, 1921, 1920.

[IKZ13] Mourad E. H. Ismail, Anisse Kasraoui, and Jiang Zeng. Separation of variables and combinatorics of linearization coefficients of orthogonal polynomials. J. Combin. Theory Ser. A, 120(3):561-599, 2013.

[ISV87] Mourad E. H. Ismail, Dennis Stanton, and Gérard Viennot. The combinatorics of $q$-Hermite polynomials and the Askey-Wilson integral. European J. Combin., 8(4):379$392,1987$.

[Jun03] Alexandre Junod. Hankel determinants and orthogonal polynomials. Expo. Math., 21(1):63-74, 2003.

[Kit07] Sergey Kitaev. Introduction to partially ordered patterns. Discrete Appl. Math., 155(8):929-944, 2007.

[Kit11] Sergey Kitaev. Patterns in Permutations and Words. Monographs in Theoretical Computer Science. Springer-Verlag, 2011.

[KLS10] Roelof Koekoek, Peter A. Lesky, and René F. Swarttouw. Hypergeometric orthogonal polynomials and their q-analogues. Springer Monographs in Mathematics. SpringerVerlag, Berlin, 2010. With a foreword by Tom H. Koornwinder.

[Knu98] Donald E. Knuth. The art of computer programming. Vol. 3. Addison-Wesley, Reading, MA, 1998. Sorting and searching, Second edition [of MR0445948].

[Kra99] C. Krattenthaler. Advanced determinant calculus. Sém. Lothar. Combin., 42:Art. B42q, 1999. The Andrews Festschrift (Maratea, 1998). 
[Kra05] C. Krattenthaler. Advanced determinant calculus: a complement. Linear Algebra Appl., 411:68-166, 2005.

[KSZ11] Anisse Kasraoui, Dennis Stanton, and Jiang Zeng. The combinatorics of Al-SalamChihara q-Laguerre polynomials. Adv. in Appl. Math., 47(2):216-239, 2011.

[KZ06] Anisse Kasraoui and Jiang Zeng. Distribution of crossings, nestings and alignments of two edges in matchings and partitions. Electron. J. Combin., 13(1):Research Paper 33, 12 pp. (electronic), 2006.

[Mac04] Percy A. MacMahon. Combinatory analysis. Vol. I, II (bound in one volume). Dover Phoenix Editions. Dover Publications Inc., Mineola, NY, 2004. Reprint of it An introduction to combinatory analysis (1920) and it Combinatory analysis. Vol. I, II (1915, 1916).

[MK15] Richard J. Martin and Michael J. Kearney. Integral representation of certain combinatorial recurrences. Combinatorica, 35(3):309-315, 2015.

[Mło10] Wojciech Młotkowski. Fuss-Catalan numbers in noncommutative probability. Doc. Math., 15:939-955, 2010.

[Mło12] Wojciech Młotkowski. Probability measures corresponding to Aval numbers. Colloq. Math., 129(2):189-202, 2012.

[MP13] Wojciech Młotkowski and Karol A. Penson. The probability measure corresponding to 2-plane trees. Probab. Math. Statist., 33(2):255-264, 2013.

[MP14] Wojciech Młotkowski and Karol A. Penson. Probability distributions with binomial moments. Infin. Dimens. Anal. Quantum Probab. Relat. Top., 17(2):1450014, 32, 2014.

[MP18] Wojciech Młotkowski and Karol A. Penson. A Fuss-type family of positive definite sequences. Colloq. Math., 151(2):289-304, 2018.

[NS06] Alexandru Nica and Roland Speicher. Lectures on the combinatorics of free probability, volume 335 of London Mathematical Society Lecture Note Series. Cambridge University Press, Cambridge, 2006.

[OEI] OEIS Foundation Inc. (2018), The On-Line Encyclopedia of Integer Sequences.

[Pos] Alex Postnikov. Total positivity, grassmannians, and networks. arXiv:math/0609764.

[Rad79] Christian Radoux. Calcul effectif de certains déterminants de Hankel. Bull. Soc. Math. Belg. Sér. B, 31(1):49-55, 1979.

[Rad90] Christian Radoux. Déterminant de Hankel construit sur les polynômes de Hermite. Ann. Soc. Sci. Bruxelles Sér. I, 104(2):59-61 (1991), 1990.

[Rad91] Christian Radoux. Déterminant de Hankel construit sur des polynômes liés aux nombres de dérangements. European J. Combin., 12(4):327-329, 1991.

[Rad92] Christian Radoux. Déterminants de Hankel et théorème de Sylvester. In Séminaire Lotharingien de Combinatoire (Saint-Nabor, 1992), volume 498 of Publ. Inst. Rech. Math. Av., pages 115-122. Univ. Louis Pasteur, Strasbourg, 1992. 
[Rad00] Christian Radoux. Addition formulas for polynomials built on classical combinatorial sequences. In Proceedings of the 8th International Congress on Computational and Applied Mathematics, ICCAM-98 (Leuven), volume 115, pages 471-477, 2000.

[Rad02] Christian Radoux. The Hankel determinant of exponential polynomials: A very short proof and a new result concerning Euler numbers. Amer. Math. Monthly, 109(3):277$278,2002$.

[Ran98] Arthur Randrianarivony. Moments des polynômes orthogonaux unitaires de Sheffer généralisés et spécialisations. European J. Combin., 19(4):507-518, 1998.

[She53] G. C. Shephard. Unitary groups generated by reflections. Canad. J. Math., 5:364-383, 1953.

[Sok18] Alan D. Sokal. The Euler and Springer numbers as moment sequences. Expo. Math., in press, 2018.

[Spe92] Roland Speicher. A noncommutative central limit theorem. Math. Z., 209(1):55-66, 1992.

[SS85] Rodica Simion and Frank W. Schmidt. Restricted permutations. European J. Combin., 6(4):383-406, 1985.

[SS94] R. Simion and D. Stanton. Specializations of generalized Laguerre polynomials. SIAM J. Math. Anal., 25(2):712-719, 1994.

[SS96] R. Simion and D. Stanton. Octabasic Laguerre polynomials and permutation statistics. J. Comput. Appl. Math., 68(1-2):297-329, 1996.

[ST43] J. A. Shohat and J. D. Tamarkin. The Problem of Moments. American Mathematical Society Mathematical surveys, vol. I. American Mathematical Society, New York, 1943.

[Ste94] Einar Steingrímsson. Permutation statistics of indexed permutations. European J. Combin., 15(2):187-205, 1994.

[SW06] Carla D. Savage and Herbert S. Wilf. Pattern avoidance in compositions and multiset permutations. Adv. in Appl. Math., 36(2):194-201, 2006.

[SW07a] John Shareshian and Michelle L. Wachs. $q$-Eulerian polynomials: excedance number and major index. Electron. Res. Announc. Amer. Math. Soc., 13:33-45, 2007.

[SW07b] Einar Steingrímsson and Lauren K. Williams. Permutation tableaux and permutation patterns. J. Combin. Theory Ser. A, 114(2):211-234, 2007.

[SZ] Alan D. Sokal and Jiang Zeng. Some multivariate master polynomials for permutations, set partitions, and perfect matchings, and their continued fractions. arXiv:2003.08192.

[Wig55] Eugene P. Wigner. Characteristic vectors of bordered matrices with infinite dimensions. Ann. of Math. (2), 62:548-564, 1955.

[Wim00] Jet Wimp. Hankel determinants of some polynomials arising in combinatorial analysis. volume 24, pages 179-193. 2000. Computational methods from rational approximation theory (Wilrijk, 1999). 